\title{
Micromotion Feature Extraction of Space Target Based on Track-Before-Detect
}

\author{
Yijun Chen, ${ }^{1,2}$ Qun Zhang, ${ }^{1,2,3}$ Ying Luo, ${ }^{1,2,3}$ and Tat Soon Yeo ${ }^{4}$ \\ ${ }^{1}$ Institute of Information and Navigation, Air Force Engineering University, Xian 710077, China \\ ${ }^{2}$ Collaborative Innovation Center of Information Sensing and Understanding, Xian 710077, China \\ ${ }^{3}$ Key Laboratory for Information Science of Electromagnetic Waves, Ministry of Education, Fudan University, Shanghai 200433, China \\ ${ }^{4}$ Department of Electrical and Computer Engineering, National University of Singapore, Singapore 11576 \\ Correspondence should be addressed to Qun Zhang; zhangqunnus@gmail.com
}

Received 29 May 2017; Accepted 16 July 2017; Published 22 August 2017

Academic Editor: Hyung-Sup Jung

Copyright ( 2017 Yijun Chen et al. This is an open access article distributed under the Creative Commons Attribution License, which permits unrestricted use, distribution, and reproduction in any medium, provided the original work is properly cited.

\begin{abstract}
The micromotion feature of space target provides an effective approach for target recognition. The existing micromotion feature extraction is implemented after target detection and tracking; thus the radar resources need to be allocated for target detection, tracking, and feature extraction, successively. If the feature extraction can be implemented by utilizing the target detecting and tracking pulses, the radar efficiency can be improved. In this paper, by establishing a feedback loop between micromotion feature extraction and track-before-detect (TBD) of target, a novel feature extraction method for space target is proposed. The TBD technology is utilized to obtain the range-slow-time curves of target scatterers. Then, micromotion feature parameters are estimated from the acquired curve information. In return, the state transition set of TBD is updated adaptively according to these extracted feature parameters. As a result, the micromotion feature parameters of space target can be extracted concurrently with implementing the target detecting and tracking. Simulation results show the effectiveness of the proposed method.
\end{abstract}

\section{Introduction}

The monitoring and recognition of space targets have become more and more important since these space targets such as fragments of satellites, rocket bodies, and other space debris are hazardous to aerospace activities with the increasing outer space explorations [1]. Generally, a space target has complex micromotion such as spinning, precession, and rolling, in addition to the body translation [2]. These micromotions will induce the micro-Doppler (m-D) effect, which can be regarded as a unique signature, providing additional information for classification, recognition, and identification of the target [3]. Therefore, since the concept of micro-Doppler was introduced to the radar signal processing field $[4,5]$, the micromotion feature extraction of space target has drawn extensive attention of scholars [6-16].

Based on the fixed scatterer model [7], the micromotion feature of space target was usually analyzed with the timefrequency distribution technique, and the micromotion feature of target was extracted with parameter transformation method [7]. However, the time-frequency methods have some limitations due to the fact that the change of microDoppler frequency with time is highly nonlinear [8]. A feature extraction method based on empirical-mode decomposition (EMD) was proposed to estimate the oscillation frequency of the truck's surface in [9]. To get the higher potential payoffs from the exploitation of $\mathrm{m}-\mathrm{D}$ effect in wideband radar system, the micromotion feature extraction for wideband radar based on complex image orthogonal matching pursuit decomposition is proposed in [10].

However, many space targets are of conical and conecylinder shapes, these rotationally symmetric targets cannot be described exactly with the fixed scatterer model. Their scattering centers of the edge of the conic bottom slide with the radar line of sight (LOS) that means the scattering centers local at the two intersections of the conic bottom edge and the radar wave incident plane [11]. Based on the slide scatterer model, the mathematical expression of microDoppler is derived, which is validated by the darkroom data in $[12,13]$. However, these works did not provide effective 
micromotion feature extraction methods for the slide scatterer model. Unfortunately, most of the existing micromotion feature extraction methods for the fixed scatterer model will not function properly because the micro-Doppler curve of a slide scatterer is much different from that of a fixed scatterer. Although some methods based on the complex generalized Radon transform and extended Hough transform are proposed for micromotion feature extraction based on the slide scatterer model $[14,15]$, the computation load of these methods is heavy, and the methods need high pulse repetition frequency ( $P R F)$ to avoid the frequency domain aliasing phenomenon. In this case, a lot of radar resources needs to be allocated for micromotion feature extraction.

In fact, the existing micromotion feature extraction methods of space target are implemented after target detection and tracking. It is necessary to allocate the limited radar resources for target detecting, tracking, and feature extraction, successively. In the case of multitarget monitoring, the allocation contradiction of radar resources will be serious. To overcome this problem, we intend to seek a novel approach which can implement target detecting and tracking simultaneously when extracting the micromotion features of a space target, which can be available for both the fixed scatterer model and the slide scatterer model.

In recent years, the track-before-detect (TBD) technology has shown good performance in weak target detecting and tracking. Unlike traditional techniques that declare the presence of a target at each scan, the TBD technology processes more consecutive scans jointly, and then it declares the presence of a target and its track [16-18]. Through the interscan accumulation, the TBD technology can improve the probability of target detection. The idea of establishing a feedback loop between feature extraction and TBD of target has been provided in our previous preliminary work [19]. In this paper, a micromotion feature extraction method of space target based on TBD technology is further proposed. In the method, the tracks of target scatterers can be obtained during the TBD of target, and the tracks are the range-slow-time curves of target scatterers indeed. We call the range-slowtime curve as "range trajectory" in this paper. On this basis, we attempt to add the micromotion feature extraction into the process of target detecting and tracking by establishing a feedback loop, namely, that the micromotion feature parameters are extracted via fitting the obtained range trajectory according to the mathematical expression of $\mathrm{m}-\mathrm{D}$ effect, and in turn the extracted micromotion feature parameters are utilized to update the parameters of TBD adaptively. As a result, the micromotion feature extraction, detecting, and tracking of space target can be implemented simultaneously with the information feedback, which can provide real-time and effective information for target recognition. By changing the mathematical expressions of different kinds of $\mathrm{m}-\mathrm{D}$ effects for range trajectory fitting, the fixed scatterer model, the slide scatterer model, and any kinds of micromotion forms can be processed with the proposed method.

This paper is organized as follows. Taking the conical target contains a fixed scatterer and two slide scatterers as an example, the micromotion feature of the target precession is analyzed in Section 2. Combining with the TBD technology,

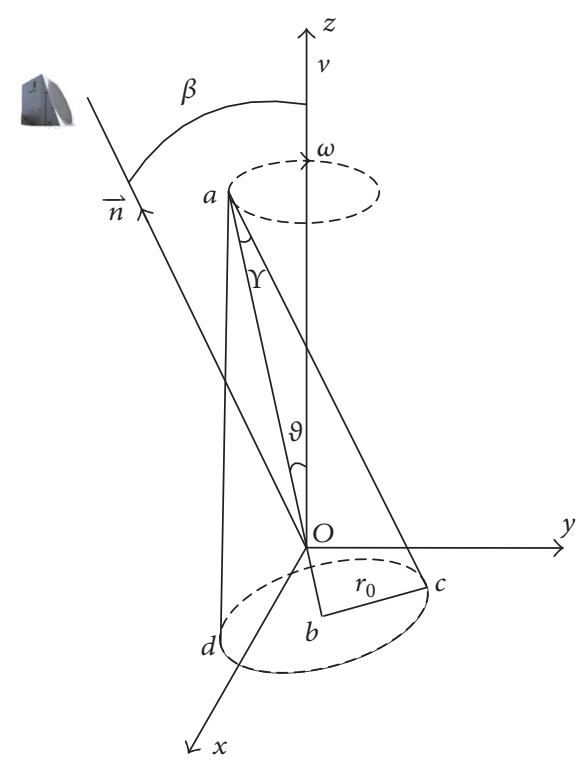

FIGURE 1: The geometry of conical target with precession.

the micromotion feature extraction method is presented in detail in Section 3. Simulations are presented in Section 4 and some conclusions are made in the last section.

\section{Micromotion Feature of Conical Target with Precession}

When the space target is flying outside the atmosphere, it needs to spin around its own axis of symmetry to maintain stability. Meanwhile, it needs to cone around a spatial directional axis due to the lateral force caused by projectile separation and bait release. These two types of rotation formed the precession of target. For a conical target, it usually consists of three dominant scatterers (i.e., one tipcone scatterer and two cone-base scatterers), where the tipcone scatterer can be treated as a fixed tip-cone scatterer, and the cone-base scatterers are slide cone-base scatterers. The geometry of conical target with precession is shown in Figure 1, where $O$ is the target center, and the target is rotating around $z$-axis with precession angle $\vartheta$ and precession frequency $\omega \mathrm{rad} / \mathrm{s}$. The distance between the radar and the target center is denoted as $R_{c}$. The radar LOS is denoted as $\overrightarrow{\mathbf{n}}, y$-axis is in the plane determined by $\overrightarrow{\mathbf{n}}$ and $z$-axis, and $x$-axis is established according to the right-hand rule. The LOS can be represented as $\overrightarrow{\mathbf{n}}=\sin \beta \overrightarrow{\mathbf{y}}+\cos \beta \overrightarrow{\mathbf{z}}$, where $\beta$ is the angle between LOS and $z$-axis. The target consists of three scatterers denoted as $a, c$, and $d$, respectively. The radius of the base circle is $r_{0}$, the distance between the target centroid and scatterer $a$ is denoted as $|o a|$, and the vertical distance between the target centroid and the bottom circle is represented as $|o b|$. According to the rigid body dynamics, the velocity of target should be along $z$-axis and the velocity is given as $v$.

Assume that the angle between $x$-axis and the projection of vector $\overrightarrow{\mathbf{o a}}$ on the $x$ oy plane is $\phi_{0}$ at time $t=0$, then the angle 
between LOS and $\overrightarrow{\mathbf{o a}}$ is denoted as $\varphi$, and it can be calculated by

$$
\cos \varphi=\sin \beta \sin \vartheta \sin \left(\omega t+\phi_{0}\right)+\cos \beta \cos \vartheta
$$

The projection of $\overrightarrow{\mathbf{o a}}, \overrightarrow{\mathbf{o c}}$, and $\overrightarrow{\mathbf{o d}}$ in the line-of-sight direction at time $t$ can be represented as $r_{a}(t), r_{c}(t)$, and $r_{d}(t)$, respectively; that is,

$$
\begin{aligned}
r_{a}(t)= & -|o a| \cdot \cos \varphi-\cos \beta \cdot v \cdot t+R_{c}=-|o a|\left(\sin \beta \sin \vartheta \sin \left(\omega t+\phi_{0}\right)+\cos \beta \cos \vartheta\right)-\cos \beta \cdot v \cdot t+R_{c} \\
r_{c}(t)= & r_{0} \cdot \sin \varphi+|o b| \cdot \cos \varphi-\cos \beta \cdot v \cdot t+R_{c} \\
= & r_{0} \sqrt{1-\cos ^{2} \beta \cos ^{2} \vartheta-\sin ^{2} \beta \sin ^{2} \vartheta \sin ^{2}\left(\omega t+\phi_{0}\right)-2 \cos \beta \cos \vartheta \sin \beta \sin \vartheta \sin \left(\omega t+\phi_{0}\right)} \\
& +|o b|\left(\sin \beta \sin \vartheta \sin \left(\omega t+\phi_{0}\right)+\cos \beta \cos \vartheta\right)-\cos \beta \cdot v \cdot t+R_{c} \\
r_{d}(t)= & -r_{0} \cdot \sin \varphi+|o b| \cdot \cos \varphi-\cos \beta \cdot v \cdot t+R_{c} \\
= & -r_{0} \sqrt{1-\cos ^{2} \beta \cos ^{2} \vartheta-\sin ^{2} \beta \sin ^{2} \vartheta \sin ^{2}\left(\omega t+\phi_{0}\right)-2 \cos \beta \cos \vartheta \sin \beta \sin \vartheta \sin \left(\omega t+\phi_{0}\right)} \\
& +|o b|\left(\sin \beta \sin \vartheta \sin \left(\omega t+\phi_{0}\right)+\cos \beta \cos \vartheta\right)-\cos \beta \cdot v \cdot t+R_{c} .
\end{aligned}
$$

Reference [1] points out that, in the wideband radar system, the micromotion feature of target can be described by range-slow-time image, where the peak of range profile appears to be a range-slow-time curve (range trajectory) which is determined by the scatterer range $r(t)$. And the range trajectory reflects the micromotion feature of target scatterer. Therefore, from (2)-(4), the micromotion feature of cone-tip scatterer $a$ is sinusoid, while that of the cone-base scatterers $c$ and $d$ is quasisinusoid, which deviates from the standard sinusoid.

During the target observation, the shielded effect should be considered. Assume that the cone half angle of target is $\Upsilon$. Obviously, $\varphi$ is changing with time $t$, and $\varphi \in[|\beta-\vartheta|, \beta+$ ๆ]. The observable scatterers are different when $\varphi$ locates in different interval range of $[0, \pi]$. Therefore, we divide $[0, \pi]$ into four observation areas as follows:

Area 1: $0 \leq \varphi<\Upsilon$, the observable scatterers are $a, c$, and $d$.

Area 2: $\Upsilon \leq \varphi<\pi / 2$, the observable scatterers are $a$ and $d$.

Area 3: $\pi / 2 \leq \varphi<\pi-\Upsilon$, the observable scatterers are $a, c$, and $d$.

Area 4: $\pi-\Upsilon \leq \varphi<\pi$, the observable scatterers are $c$ and $d$.

The impact of the shielded effect on the proposed micromotion feature extraction method will be discussed in Section 3.

Next, a simple simulation is given to validate the correctness of theoretical analysis. The pulse duration is $T_{p}=$ $1 \mu \mathrm{s}$, carrier frequency is $f_{c}=10 \mathrm{GHz}$, signal bandwidth is $B=3 \mathrm{GHz}$, pulse repetition frequencies is $\mathrm{PRF}=60 \mathrm{~Hz}$, and the coherent processing time is $T_{c}=1 \mathrm{~s}$. The radius of the base circle is $r_{0}=1 \mathrm{~m}$, the distance between the target center and cone-tip scatterer $a$ is $|o a|=3 \mathrm{~m}$, and the vertical distance between the target center and the bottom circle is $|o b|=0.3 \mathrm{~m}$.
To observe the micromotion feature of target more intuitively, we assume that $R_{c}=0 \mathrm{~m}$ and $v=0 \mathrm{~m} / \mathrm{s}$. The precession angle is $\vartheta=15^{\circ}$, precession frequency is $\omega=8 \pi \mathrm{rad} / \mathrm{s}$, the angle between LOS and $z$-axis is $\beta=145^{\circ}$, and the initial angle is $\phi_{0}=\pi / 100 \mathrm{rad}$. We can obtain the range-slow-time image as shown in Figure 2.

From Figure 2, we can see that the range profile peak of scatterer $a$ changes with slow-time following the sinusoidal form, while those of the sliding scatterers $c$ and $d$ are quasisinusoids (deviating from the standard sinusoid form), which confirms the theoretical analysis.

\section{Micromotion Feature Extraction Based on TBD}

Due to the requirement of additional observation pulses after target detection and tracking, the existing micromotion feature extraction methods usually occupy much radar resources. Also, the real-time performance and radar efficiency are not satisfied. So we intend to combine the micromotion feature extraction with target detecting and tracking in the way of information feedback and then implement the micromotion feature extraction, detecting and tracking of space target simultaneously. It will save the radar resources and improve the radar efficiency and real-time performance of micromotion feature extraction.

In this section, we establish a feedback loop between micromotion feature extraction and TBD of target. Firstly, based on TBD technology, the target scatterer range trajectory information is backtracked along with the energy accumulation process, and then the micromotion feature parameters of target can be fitted with these obtained trajectory information. In return, the extracted micromotion feature parameters are used to update the state transition set of TBD adaptively, and the results of micromotion feature extraction are considered into the declaration of the target presence. As a result, micromotion feature extraction, target detecting, and tracking can be completed simultaneously. 


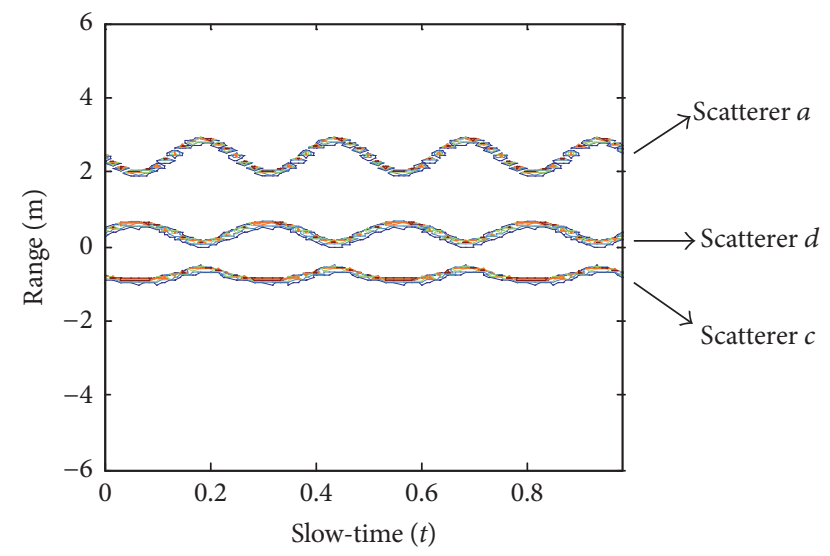

FIgURE 2: Range-slow-time image of precession target.

3.1. Range Trajectory Backtracking and Micromotion Feature Extraction. For simpleness, the range trajectory backtracking for single scatterer based on TBD is described firstly. The monitoring area is divided into $N_{r} \times N_{\theta}$ grids according to the range and azimuthal angle, and each grid is denoted as a state $(i, j), i=1, \ldots, N_{r}, j=1, \ldots, N_{\theta}$, which represents the position $\left(\left(i-N_{r} / 2\right) \cdot \Delta r+R_{0},\left(j-N_{\theta} / 2\right) \Delta \theta+\theta_{0}\right)$, where $\Delta r$ and $\Delta \theta$ are the stepped increasement of the range and azimuthal angle, and $\left(R_{0}, \theta_{0}\right)$ is the center of the monitoring area. Assume the radar transmits wideband signal $p(\widehat{t})$. At each scan, the beams towards all the azimuthal angles $\left(N_{\theta}\right.$ angles) are formed by the radar. The beam width is denoted as $\phi_{B}$, each state $(i, j)$ will be hit by $M+1$ successive beams, where

$$
M= \begin{cases}\left\lfloor\frac{\phi_{B}}{\Delta \theta}\right\rfloor, & \left\lfloor\frac{\phi_{B}}{\Delta \theta}\right\rfloor \text { is even } \\ \left\lfloor\frac{\phi_{B}}{\Delta \theta}\right\rfloor-1, & \left\lfloor\frac{\phi_{B}}{\Delta \theta}\right\rfloor \text { is odd. }\end{cases}
$$

Obviously, the sequence number of these $M+1$ beams should be $(j-M / 2, j-M / 2+1, \ldots, j+M / 2)$.

At the $k$ th scan, the echo signal of the state $\mathbf{x}_{k}=\left(i_{k}, j_{k}\right)$ of the $m$ th beam can be represented as

$$
\begin{aligned}
s_{k, m, \mathbf{x}_{k}}(\hat{t})= & \sigma_{k\left(i_{k}, j_{k}\right)} G_{m}\left(j_{k}\right) \\
& \cdot p\left(\widehat{t}-\frac{2\left(i_{k}-N_{r} / 2\right) \cdot \Delta r+2 R_{0}}{c}\right), \\
& \hat{t} \in\left[-\frac{T_{p}}{2}, \frac{T_{p}}{2}\right], m \in(1, \ldots, M+1),
\end{aligned}
$$

where $G_{m}(\cdot)$ represents the beampattern of the $m$ th beam and $G_{m}\left(j_{k}\right)$ represents the obtained transmit gain at the $j_{k}$ th azimuthal angle with the $m$ th beam and $\sigma_{k\left(i_{k}, j_{k}\right)}$ is the backscattered amplitude of the state $\left(i_{k}, j_{k}\right)$ at the $k$ th scan.
After performing range compression, the high-resolution range profile (HRRP) can be obtained as

$$
\begin{aligned}
S_{k, m, \mathbf{x}_{k}} & \left(F_{f}\right) \\
= & T_{p} \cdot G_{m}\left(j_{k}\right) \cdot \sigma_{k\left(i_{k}, j_{k}\right)} \\
& \cdot \operatorname{sinc}\left(F_{f}+\frac{2\left(i_{k}-N_{r} / 2\right) \cdot \Delta r+2 R_{0}}{c}\right) .
\end{aligned}
$$

Thus, at the $k$ th scan, the measured value of each state $\mathbf{x}_{k}=\left(i_{k}, j_{k}\right)$ can be defined as

$$
\begin{array}{r}
Z_{k}\left(\mathbf{x}_{k}\right)=\left.\sum_{m=1}^{M+1}\left|S_{k, m, \mathbf{x}_{k}}\left(F_{f}\right)\right|_{F_{f}=-\left(2\left(i_{k}-N_{r} / 2\right) \cdot \Delta r+2 R_{0}\right) / c}\right|^{2}, \\
i_{k}=1, \ldots, N_{r}, j_{k}=1, \ldots, N_{\theta} .
\end{array}
$$

At the $k$ th scan, the cumulative energy of state $\mathbf{x}_{k}$ is denoted as $I\left(\mathbf{x}_{k}\right)$, and it can be calculated as

$$
I\left(\mathbf{x}_{k}\right)=Z_{k}\left(\mathbf{x}_{k}\right)+\max _{\mathbf{x}_{k-1} \in \Gamma\left(\mathbf{x}_{k}\right)}\left(I\left(\mathbf{x}_{k-1}\right)\right)
$$

where $\Gamma\left(\mathbf{x}_{k}\right)$ is the state transition set. $\Gamma\left(\mathbf{x}_{k}\right)$ contains all the possible state $\mathbf{x}_{k-1}$ which can transit to state $\mathbf{x}_{k}$. Set an appropriate detection threshold for the cumulative energy function $I\left(\mathbf{x}_{k}\right)$ after $K$ scans accumulation, and the state sequence whose cumulative energy is larger than the threshold can be backtracked according to

$$
B_{k}\left(\mathbf{x}_{k}\right)=\arg \max _{\mathbf{x}_{k-1} \in \Gamma\left(\mathbf{x}_{k}\right)}\left(I\left(\mathbf{x}_{k-1}\right)\right),
$$

where $B_{k}\left(\mathbf{x}_{k}\right)$ is the backtracking function, which is used to record the state corresponding to the maximum cumulative energy at each scan. Assume that the recorded state of the $k$ th scan is $\mathbf{x}_{k}=\left(i_{k}, j_{k}\right)$, the estimated scatterer range trajectory can be denoted as $R(k)=\left(i_{k}-N_{r} / 2\right) \cdot \Delta r+R_{0}, k=1, \ldots, K$, and the angle trajectory is $\Theta(k)=\left(j_{k}-N_{\theta} / 2\right) \Delta \theta+\theta_{0}, k=1, \ldots, K$.

For the cone-tip scatterer $a$, the estimated range trajectory

$$
R(k)=r_{a}(k \cdot \Delta t)
$$

should be equal to (2), where $\Delta t$ is the time interval between the two adjacent scans. Without loss of generality, it holds $\Delta t=1 /$ PRF. Similarly, for the cone-base scatterers $c$ and $d$, the estimated range trajectories $R(k)$ should be equal to (3) and (4), respectively.

However, the TBD method described above can obtain only one scatterer range trajectory, which cannot meet the requirement of getting range trajectories of each scatterers. Although some TBD methods for multitarget detecting and tracking have been proposed $[20,21]$, they require that the state of different targets can not be the same. However, in this paper, the different scatterers are usually at the same azimuthal angle and may be of the same range at some scans (i.e., the range trajectories may be intersected). That means the different scatterers may have the same state, which leads to the existing method which can not be used. To resolve this problem, we improve the TBD method as follows. 
Assume the target consists of $P$ observable scatterers. At the $k$ th scan, for each azimuthal angle $j_{k}$, select any $P$ states to form an expanded state $\left(\left(i_{k, 1}, j_{k}\right),\left(i_{k, 2}, j_{k}\right), \ldots,\left(i_{k, P}, j_{k}\right)\right)$, where the $P$ states can be the same; that is, $i_{k, p 1}$ can be equal to $i_{k, p 2}$ when $p_{1} \neq p_{2}$. The measured value of the expanded state $\mathbf{y}_{k}=\left\{\left(i_{k, 1}, j_{k}\right),\left(i_{k, 2}, j_{k}\right), \ldots,\left(i_{k, P}, j_{k}\right)\right\}$ is defined as

$$
Z_{k}\left(\mathbf{y}_{k}\right)=\sum_{p=1}^{P} Z_{k}\left(\left(i_{k, p}, j_{k}\right)\right)
$$

On the basis, the cumulative energy shown as (9) is conducted in terms of the expanded state, where the state correlation is necessary. For example, there are 6 kinds of states correlation approaches when $P=3$, shown as Figure 3.

Among these 6 kinds of states correlation approaches, only one is consistent with the actual scatterers trajectories. In [22], we have proposed a state correlation approach which can be used for states correlation.

After $K$ scans, the cumulative energy $I\left(\mathbf{y}_{k}\right)$ should be compared with a detection threshold $T_{\alpha}$ :

$$
T_{\alpha}=\gamma K P,
$$

where $\gamma$ is a constant which will affect the target detecting performance, in this paper we call $\gamma$ as "detection threshold coefficient." The state sequences whose cumulative energy is larger than the threshold can be backtracked according to (10).

It should be pointed out that the states correlation leads to the dependence of the energy accumulation of each scatterer. A specific expression of the detection threshold coefficient is difficult to be derived with a given false alarm probability. Just as [20], the detection threshold coefficient can be chosen from Monte-Carlo experiments.

Assume that the number of the cumulative energy $I\left(\mathbf{y}_{k}\right)$ which is larger than the threshold is $Q$. Thus, $Q$ state sequences will be obtained, and each state sequence contains $P$ range trajectories $R_{p}(k)=\left(i_{k}-N_{r} / 2\right) \cdot \Delta r+R_{0}(p=$ $1, \ldots, P, k=1, \ldots, K)$ and $P$ angle trajectories $\Theta_{p}(k)=$ $\left(j_{k}-N_{\theta} / 2\right) \Delta \theta+\theta_{0}(p=1, \ldots, P, k=1, \ldots, K)$. Due to the fact that intersections number of any two range trajectories will be small, a condition for selecting the reasonable state sequence from the $Q$ state sequences is defined as

$$
C\left(R_{p 1}(k)=R_{p 2}(k)\right)<\varsigma K, \quad \forall p 1 \neq p 2,
$$

where $C\left(R_{p 1}(k)=R_{p 2}(k)\right)$ represents the intersections number of $R_{p 1}(k)$ and $R_{p 2}(k)$ and $\varsigma$ is a constant used to control the ratio of intersections number to scans number $K$. The state sequences which satisfy the condition shown as (14) are selected, and the corresponding range trajectories and angle trajectories are obtained from the target scatterers.

For each possible value of $P$, the TBD procedure proposed above can be conducted to obtain the range trajectories of $P$ scatterers. The shielded effect has been discussed in Section 2: when $\varphi$ falls with in Area 1 or Area 3, three scatterers are observable (corresponding to $P=3$ ); when $\varphi$ falls within Area 2 or Area 4, two scatterers are observable (corresponding to $P=2$ ). Therefore, the possible values of $P$ are $P=3$ and $P=2$. Thus, the TBD procedure of $P=3$ and $P=2$ should be conducted.

In the case of $P=3$, if there is state sequence which satisfies (14) which can be selected out, three range trajectories (i.e., $R_{1}(k), R_{2}(k)$, and $R_{3}(k)$ ) can be obtained. Firstly, we assume that $R_{1}(k)$ is the range trajectory of the cone-tip scatterer $a$ and $R_{2}(k)$ and $R_{3}(k)$ are the range trajectories of the cone-base scatterers $c$ and $d$, respectively. $R_{1}(k), R_{2}(k)$, and $R_{3}(k)$ can be represented as

$$
\begin{aligned}
& R_{1}(k)=r_{a}(k \cdot \Delta t)+\varepsilon(k), \\
& R_{2}(k)=r_{c}(k \cdot \Delta t)+\varepsilon(k), \\
& R_{3}(k)=r_{d}(k \cdot \Delta t)+\varepsilon(k),
\end{aligned}
$$

where $\varepsilon(k)$ represents the error induced from the noise and TBD procedure. We can estimate the micromotion feature parameter vector $\mathbf{P}_{\mathbf{A}}=\left[|o a|, \phi_{0}, \beta, \vartheta, \omega, v,|o b|, r_{0}, R_{c}\right]$ by fitting $R_{1}(k), R_{2}(k)$, and $R_{3}(k)$ according to the curve form of standard sinusoid shown as (2) and quasisinusoid shown as (3)-(4) with the least squares method:

$$
\begin{aligned}
\min _{\mathbf{P}_{\mathrm{A}}} & \left\|R_{1}(k)+|o a|\left(\sin \beta \sin \vartheta \sin \left(\omega k \cdot \Delta t+\phi_{0}\right)+\cos \beta \cos \vartheta\right)+\cos \beta \cdot v k \cdot \Delta t-R_{c}\right\|+\| R_{2}(k) \\
& -r_{0} \sqrt{1-\cos ^{2} \beta \cos ^{2} \vartheta-\sin ^{2} \beta \sin ^{2} \vartheta \sin ^{2}\left(\omega k \cdot \Delta t+\phi_{0}\right)-2 \cos \beta \cos \vartheta \sin \beta \sin \vartheta \sin \left(\omega k \cdot \Delta t+\phi_{0}\right)} \\
& -|o b|\left(\sin \beta \sin \vartheta \sin \left(\omega k \cdot \Delta t+\phi_{0}\right)+\cos \beta \cos \vartheta\right)+\cos \beta \cdot v k \cdot \Delta t-R_{c}\|+\| R_{3}(k) \\
& +r_{0} \sqrt{1-\cos ^{2} \beta \cos ^{2} \vartheta-\sin ^{2} \beta \sin ^{2} \vartheta \sin ^{2}\left(\omega k \cdot \Delta t+\phi_{0}\right)-2 \cos \beta \cos \vartheta \sin \beta \sin \vartheta \sin \left(\omega k \cdot \Delta t+\phi_{0}\right)} \\
& -|o b|\left(\sin \beta \sin \vartheta \sin \left(\omega k \cdot \Delta t+\phi_{0}\right)+\cos \beta \cos \vartheta\right)+\cos \beta \cdot v k \cdot \Delta t-R_{c} \| \cdot
\end{aligned}
$$

Equation (16) can be solved with the LevenbergMarquardt method [23], which is sensitive to the initial values. Therefore, how to set the appropriate initial values is proposed as follows. The EMD method [24] can be used to 

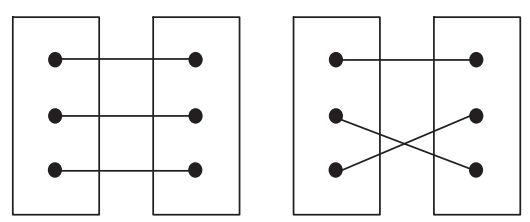
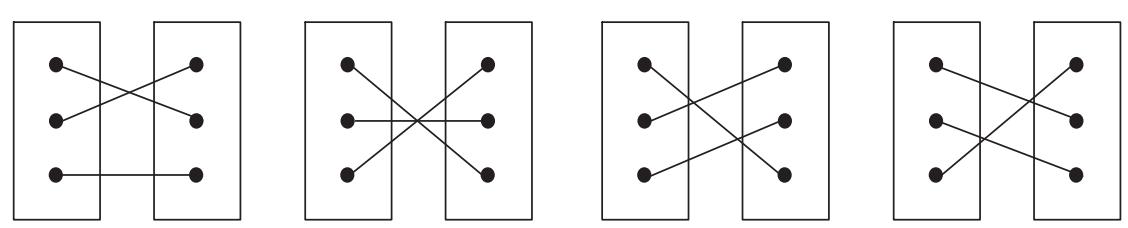

FIGURE 3: State correlation approaches.

separate $R_{1}(k)$ into a set of intrinsic mode functions (IMF) which is descended by frequency. Thus, we can obtain

$$
\begin{aligned}
I_{1, R_{1}}(k) & =B_{a}+C_{a} k, \\
I_{2, R_{1}}(k) & =A_{a} \sin \left(\omega_{a} k+\phi_{0_{a}}\right) \\
A_{a} & =-|o a| \sin \beta \sin \vartheta+\xi,
\end{aligned}
$$

$$
\begin{aligned}
B_{a} & =-|o a| \cos \beta \cos \vartheta+R_{c}+\xi \\
C_{a} & =-\cos \beta \cdot v+\xi \\
\omega_{a} & =\omega+\xi \\
\phi_{0_{a}} & =\phi_{0}+\xi
\end{aligned}
$$

where $\xi$ is the error from $\varepsilon(k)$ and EMD method. According to (3) and (4), we can get

$$
\begin{aligned}
R_{+}(k) & =R_{2}(k)+R_{3}(k)=2|o b|\left(\sin \beta \sin \vartheta \sin \left(\omega k+\phi_{0}\right)+\cos \beta \cos \vartheta\right)-2 \cos \beta \cdot v k+2 R_{c}+\varepsilon(k) \\
R_{-}(k) & =R_{2}(k)-R_{3}(k) \\
& =2 r_{0} \sqrt{1-\cos ^{2} \beta \cos ^{2} \vartheta-\sin ^{2} \beta \sin ^{2} \vartheta \sin ^{2}\left(\omega k \cdot \Delta t+\phi_{0}\right)-2 \cos \beta \cos \vartheta \sin \beta \sin \vartheta \sin \left(\omega k \cdot \Delta t+\phi_{0}\right)}+\varepsilon(k) .
\end{aligned}
$$
obtain

Similarly, separating $R_{+}(k)$ with the EMD method, we can

$$
\begin{aligned}
I_{1, R_{+}}(k) & =B_{c d}+C_{c d} k, \\
I_{2, R_{+}}(k) & =A_{c d} \sin \left(\omega_{c d} k+\phi_{0_{c d}}\right) \\
A_{c d} & =2|o b| \sin \beta \sin \vartheta+\xi, \\
B_{c d} & =2|o b| \cos \beta \cos \vartheta+2 R_{c}+\xi,
\end{aligned}
$$

$$
\begin{aligned}
C_{c d} & =-2 \cos \beta \cdot v+\xi, \\
\omega_{c d} & =\omega+\xi, \\
\phi_{0_{c d}} & =\phi_{0}+\xi .
\end{aligned}
$$

The initial values $|o a|_{\text {ini }}, \phi_{\text {oini }}, \beta_{\text {ini }}, \vartheta_{\text {ini }}, \omega_{\text {ini }}, v_{\text {ini }},|o b|_{\text {ini }}$, $r_{0 \text { ini }}$, and $R_{c \text { ini }}$ for (19) can be obtained according the equation set:

$-|o a|_{\text {ini }} \cos \beta_{\text {ini }} \cos \vartheta_{\text {ini }}+R_{c \text { ini }}=I_{1, R_{1}}(0)$

$2|o b|_{\text {ini }} \cos \beta_{\text {ini }} \cos \vartheta_{\text {ini }}+2 R_{\text {cini }}=I_{1, R_{+}}(0)$

$\cos \beta_{\text {ini }} \cdot v_{\text {ini }}=-\frac{\left(\left(I_{1, R_{1}}\left(k_{2}\right)-I_{1, R_{1}}\left(k_{1}\right)\right) /\left(k_{2}-k_{1}\right)+\left(I_{1, R_{+}}\left(k_{2}\right)-I_{1, R_{+}}\left(k_{1}\right)\right) /\left(k_{2}-k_{1}\right)\right)}{2}$

$\left.|| o a\right|_{\text {ini }} \sin \beta_{\text {ini }} \sin \vartheta_{\text {ini }} \mid=\frac{\max \left(I_{2, R_{1}}\right)-\min \left(I_{2, R_{1}}\right)}{2}$

$\left.|2| o b\right|_{\text {ini }} \sin \beta_{\text {ini }} \sin \vartheta_{\text {ini }} \mid=\frac{\max \left(I_{1, R_{+}}\right)-\min \left(I_{1, R_{+}}\right)}{2}$ 


$$
\begin{aligned}
& \phi_{\text {oini }} \\
& =\left\{\begin{array}{l}
\frac{\left(\operatorname{ar} \sin \left(I_{2, R_{1}}(0) /-\left.|| o a\right|_{\text {ini }} \sin \beta_{\text {ini }} \sin \vartheta_{\text {ini }} \mid\right)+\operatorname{arsin}\left(I_{2, R_{+}}(0) /\left.|2| o b\right|_{\text {ini }} \sin \beta_{\text {ini }} \sin \vartheta_{\text {ini }} \mid\right)\right)}{2}, \quad \sin \beta_{\text {ini }} \sin \vartheta_{\text {ini }}>0 \\
\frac{\left(\operatorname{arsin}\left(I_{2, R_{1}}(0) /\left.|| o a\right|_{\text {ini }} \sin \beta_{\text {ini }} \sin \vartheta_{\text {ini }} \mid\right)+\operatorname{arsin}\left(I_{2, R_{+}}(0) /-\left.|2| o b\right|_{\text {ini }} \sin \beta_{\text {ini }} \sin \vartheta_{\text {ini }} \mid\right)\right)}{2}, \quad \sin \beta_{\text {ini }} \sin \vartheta_{\text {ini }}<0
\end{array}\right. \\
& \omega_{\text {ini }}=\frac{\left(\arg \max _{\omega}\left(\operatorname{FFT}\left(I_{2, R_{1}}\right)\right)+\arg \max _{\omega}\left(\operatorname{FFT}\left(I_{2, R_{+}}(k)\right)\right)\right)}{2} \\
& 2 r_{0 \text { ini }} \sqrt{1-\cos ^{2} \beta_{\text {ini }} \cos ^{2} \vartheta_{\text {ini }}-\sin ^{2} \beta_{\text {ini }} \sin ^{2} \vartheta_{\text {ini }} \sin ^{2}\left(\phi_{0 \text { ini }}\right)-2 \cos \beta_{\text {ini }} \cos \vartheta_{\text {ini }} \sin \beta_{\text {ini }} \sin \vartheta_{\text {ini }} \sin \left(\phi_{0 \text { ini }}\right)}=R_{-}(0),
\end{aligned}
$$

where $\operatorname{ar} \sin (\cdot)$ is the inverse function of $\sin (\cdot)$. In $(20)$, the equations' number is one less than unknown parameters number. Thus, we defined the search interval and the search stepped increasement of $r_{0}$ as $\left[r_{\min }, r_{\max }\right]$ and $\Delta r_{0}$, respectively. For each initial values $r_{0 \text { ini }}\left(s_{n}\right)=r_{\min }+$ $\left(s_{n}-1\right) \cdot \Delta r_{0}, s_{n}=1,2, \ldots, S_{N}$, and $S_{N}=\left(r_{\max }-\right.$ $\left.r_{\text {min }}\right) / \Delta r_{0}$, a group of initial values of $|o a|_{\text {ini }}\left(s_{n}\right), \phi_{0 \text { ini }}\left(s_{n}\right)$, $\beta_{\text {ini }}\left(s_{n}\right), \vartheta_{\text {ini }}\left(s_{n}\right), \omega_{\text {ini }}\left(s_{n}\right), v_{\text {ini }}\left(s_{n}\right),|o b|_{\text {ini }}\left(s_{n}\right)$, and $R_{c \text { ini }}\left(s_{n}\right)$ can be obtained according to (20). Based on the initial values, the corresponding micromotion feature parameter vector $\mathbf{P}_{\mathbf{A}}\left(s_{n}\right)=\left[|o a|\left(s_{n}\right), \phi_{0}\left(s_{n}\right), \beta\left(s_{n}\right), \vartheta\left(s_{n}\right), \omega\left(s_{n}\right), v\left(s_{n}\right),|o b|\left(s_{n}\right)\right.$, $\left.r_{0}\left(s_{n}\right), R_{c}\left(s_{n}\right)\right]$ can be obtained by solving (16) with the Levenberg-Marquardt method. The fitting error with the $s_{n}$ th group initial values is calculated as

$$
\begin{aligned}
& E\left(s_{n}\right)=\frac{1}{3 K}\left(\left\|R_{1}(k)+|o a|\left(s_{n}\right) F_{1}\left(s_{n}\right)\right\|\right. \\
& +\left\|R_{2}(k)-r_{0}\left(s_{n}\right) \sqrt{F_{2}\left(s_{n}\right)}-|o b|\left(s_{n}\right) F_{1}\left(s_{n}\right)\right\| \\
& \left.+\left\|R_{3}(k)+r_{0}\left(s_{n}\right) \sqrt{F_{2}\left(s_{n}\right)}-|o b|\left(s_{n}\right) F_{1}\left(s_{n}\right)\right\|\right), \\
& F_{1}\left(s_{n}\right)=\sin \beta\left(s_{n}\right) \sin \vartheta\left(s_{n}\right) \sin \left(\omega\left(s_{n}\right) k+\phi_{0}\left(s_{n}\right)\right) \\
& +\cos \beta\left(s_{n}\right) \cos \vartheta\left(s_{n}\right)+\cos \beta\left(s_{n}\right) \cdot v\left(s_{n}\right) k \\
& -R_{c}\left(s_{n}\right), \\
& F_{2}\left(s_{n}\right)=1-\cos ^{2} \beta\left(s_{n}\right) \cos ^{2} \vartheta\left(s_{n}\right)-\sin ^{2} \beta\left(s_{n}\right) \\
& \cdot \sin ^{2} \vartheta\left(s_{n}\right) \sin ^{2}\left(\omega\left(s_{n}\right) k+\phi_{0}\left(s_{n}\right)\right)-2 \cos \beta\left(s_{n}\right) \\
& \cdot \cos \vartheta\left(s_{n}\right) \sin \beta\left(s_{n}\right) \sin \vartheta\left(s_{n}\right) \\
& \cdot \sin \left(\omega\left(s_{n}\right) k+\phi_{0}\left(s_{n}\right)\right) \text {. }
\end{aligned}
$$

All $E\left(s_{n}\right)$ is compared with each other, and the estimated micromotion feature parameter vector is defined as

$$
\widehat{\mathbf{P}_{\mathbf{A}}} \triangleq \mathbf{P}_{\mathbf{A}}\left(\arg \min _{s_{n}} E\left(s_{n}\right)\right)
$$

The fitting error is denoted as

$$
\widehat{E} \triangleq \min _{s_{n}} E\left(s_{n}\right)
$$

It should be pointed that $\widehat{\mathbf{P}_{\mathrm{A}}}$ and $\widehat{E}$ are obtained under the assumption that $R_{1}(k)$ is the range trajectory of the cone-tip scatterer $a$. Therefore, we rewrite them as $\widehat{\mathbf{P}_{\mathbf{A}}}\left(R_{1}\right)$ and $\widehat{E}\left(R_{1}\right)$.

Next, we assume $R_{2}(k)$ and $R_{3}(k)$ are the range trajectory of the cone-tip scatterer, respectively. The micromotion feature parameter vectors $\widehat{\mathbf{P}_{\mathbf{A}}}\left(R_{2}\right)$ and $\widehat{\mathbf{P}_{\mathbf{A}}}\left(R_{3}\right)$ can be obtained, and the corresponding fitting errors are denoted as $\widehat{E}\left(R_{2}\right)$ and $\widehat{E}\left(R_{3}\right)$. The micromotion feature parameter vector corresponding to the minimum fitting error is selected out as the final micromotion feature parameter vector

$$
\widehat{\mathbf{P}_{\mathbf{A} f}} \triangleq \widehat{\mathbf{P}_{\mathbf{A}}}\left(\arg \min _{R_{1}, R_{2}, R_{3}} \widehat{E}\right)
$$

The fitting error is denoted as

$$
\widehat{E}_{f} \triangleq \min _{R_{1}, R_{2}, R_{3}} \widehat{E}
$$

Similarly, in the case of $P=2$, the micromotion feature parameter vector can be obtained. What should be pointed out is that if the two range trajectories are corresponding to the cone-base scatterers $c$ and $d$, respectively, the parameter $|o a|$ can not be obtained.

3.2. Adaptive Update of State Transition Set. Just as mentioned in (18), the state transition set contains all the possible state $\mathbf{y}_{k-1}$ which can transit to state $\mathbf{y}_{k}$, and the construction of state transition set will significantly affect the efficiency and 
performance of TBD and micromotion feature extraction. In this paper, the state transition set is updated adaptively, by forecasting the state of scatterers in next scan according to the extracted micromotion feature parameters.

Assume that, in the $k$ th scan, the cumulative energy $I\left(\mathbf{y}_{k}\right)$ is larger than the threshold; the extracted micromotion feature parameter vector can be obtained with the method proposed in Section 3.1, which is represented as

$$
\begin{aligned}
& \widehat{\mathbf{P}_{\mathbf{A} f, \mathbf{y}_{k}}} \\
& \quad=\left[|o a|_{\mathbf{y}_{k}}, \phi_{0 \mathbf{y}_{k}}, \beta_{\mathbf{y}_{k}}, \vartheta_{\mathbf{y}_{k}}, \omega_{\mathbf{y}_{k}}, v_{\mathbf{y}_{k}},|o b|_{\mathbf{y}_{k}}, r_{0 \mathbf{y}_{k}}, R_{c \mathbf{y}_{k}}\right] .
\end{aligned}
$$

The corresponding fitting error is denoted as $\widehat{E}_{f, \mathbf{y}_{k}}$.

The state transition set can be determined in light of the extracted micromotion feature parameters. The state $\mathbf{y}_{k}$ will belong to the state transition set $\Gamma\left(\mathbf{y}_{k+1}\right)$ (i.e., $\mathbf{y}_{k} \in \Gamma\left(\mathbf{y}_{k+1}\right)$ ) when it satisfies

$$
\begin{aligned}
\left|\left(i_{k+1,1}-\frac{N_{r}}{2}\right) \cdot \Delta r+R_{0}-\Delta r_{a}\right| & <f_{R}\left(C_{s, \mathbf{y}_{k}}\right) \cdot \Delta r, \\
\left|j_{k+1}-j_{k}\right| & <2,
\end{aligned}
$$

for scatterer $a$

$$
\begin{gathered}
\left|\left(i_{k+1,2}-\frac{N_{r}}{2}\right) \cdot \Delta r+R_{0}-\Delta r_{c}\right|<f_{R}\left(C_{s, \mathbf{y}_{k}}\right) \cdot \Delta r, \\
\left|j_{k+1}-j_{k}\right|<2,
\end{gathered}
$$

for scatterer $c$

$$
\begin{aligned}
\left|\left(i_{k+1,3}-\frac{N_{r}}{2}\right) \cdot \Delta r+R_{0}-\Delta r_{d}\right| & <f_{R}\left(C_{s, \mathbf{y}_{k}}\right) \cdot \Delta r, \\
\left|j_{k+1}-j_{k}\right| & <2,
\end{aligned}
$$

for scatterer $d$,

where

$$
\begin{aligned}
& \Delta r_{a}=-|o a|_{\mathbf{y}_{k}}\left(\sin \beta_{\mathbf{y}_{k}} \sin \vartheta_{\mathbf{y}_{k}} \sin \left(\omega_{\mathbf{y}_{k}}(k+1)+\phi_{0 \mathbf{y}_{k}}\right)+\cos \beta_{\mathbf{y}_{k}} \cos \vartheta_{\mathbf{y}_{k}}\right)-\cos \beta_{\mathbf{y}_{k}} v_{\mathbf{y}_{k}}(k+1)+R_{c \mathbf{y}_{k}} \\
& \Delta r_{c} \\
& =r_{0 \mathbf{y}_{k}} \sqrt{1-\cos ^{2} \beta_{\mathbf{y}_{k}} \cos ^{2} \vartheta_{\mathbf{y}_{k}}-\sin ^{2} \beta_{\mathbf{y}_{k}} \sin ^{2} \vartheta_{\mathbf{y}_{k}} \sin ^{2}\left(\omega_{\mathbf{y}_{k}}(k+1)+\phi_{0 \mathbf{y}_{k}}\right)-2 \cos \beta_{\mathbf{y}_{k}} \cos \vartheta_{\mathbf{y}_{k}} \sin \beta_{\mathbf{y}_{k}} \sin \vartheta_{\mathbf{y}_{k}} \sin \left(\omega_{\mathbf{y}_{k}}(k+1)+\phi_{0 \mathbf{y}_{k}}\right)} \\
& +|o b|_{\mathbf{y}_{k}}\left(\sin \beta_{\mathbf{y}_{k}} \sin \vartheta_{\mathbf{y}_{k}} \sin \left(\omega_{\mathbf{y}_{k}}(k+1)+\phi_{0 \mathbf{y}_{k}}\right)+\cos \beta_{\mathbf{y}_{k}} \cos \vartheta_{\mathbf{y}_{k}}\right)-\cos \beta_{\mathbf{y}_{k}} \cdot v_{\mathbf{y}_{k}}(k+1)+R_{c \mathbf{y}_{k}} \text {. } \\
& =-r_{0 \mathbf{y}_{k}} \sqrt{1-\cos ^{2} \beta_{\mathbf{y}_{k}} \cos ^{2} \vartheta_{\mathbf{y}_{k}}-\sin ^{2} \beta_{\mathbf{y}_{k}} \sin ^{2} \vartheta_{\mathbf{y}_{k}} \sin ^{2}\left(\omega_{\mathbf{y}_{k}}(k+1)+\phi_{0 \mathbf{y}_{k}}\right)-2 \cos \beta_{\mathbf{y}_{k}} \cos \vartheta_{\mathbf{y}_{k}} \sin \beta_{\mathbf{y}_{k}} \sin \vartheta_{\mathbf{y}_{k}} \sin \left(\omega_{\mathbf{y}_{k}}(k+1)+\phi_{0 \mathbf{y}_{k}}\right)} \\
& +|o b|_{\mathbf{y}_{k}}\left(\sin \beta_{\mathbf{y}_{k}} \sin \vartheta_{\mathbf{y}_{k}} \sin \left(\omega_{\mathbf{y}_{k}}(k+1)+\phi_{0 \mathbf{y}_{k}}\right)+\cos \beta_{\mathbf{y}_{k}} \cos \vartheta_{\mathbf{y}_{k}}\right)-\cos \beta_{\mathbf{y}_{k}} \cdot v_{\mathbf{y}_{k}}(k+1)+R_{c, \mathbf{y}_{k}}
\end{aligned}
$$

$C_{s, \mathbf{y}_{k}}$ represents the consistency of the micromotion feature parameter vectors between $k-1$ th scan and $k$ th scan, and $f_{R}(\cdot)$ is an adaptive adjustment function which is used to control the size of searching range gate during TBD according to $C_{s, \mathbf{y}_{k}}$. In this paper, $C_{s, \mathbf{y}_{k}}$ is defined as

$$
C_{s, \mathbf{y}_{k}}=\operatorname{mean}\left(\frac{\left|\widehat{\mathbf{P}_{\mathbf{A} f, \mathbf{y}_{k}}}-\widehat{\mathbf{P}_{\mathbf{A} f, \mathbf{y}_{k-1}}}\right|}{\left|\widehat{\mathbf{P}}_{\mathbf{A} f, \mathbf{y}_{k-1}}\right|}\right),
$$

where mean $(\cdot)$ represents the mean value of a vector. Obviously, the smaller $C_{s, \mathbf{y}_{k}}$ is, the higher consistency is.

Obviously, if $C_{s, \mathbf{y}_{k}}$ is relatively small, it shows that the consistency of the extracted micromotion feature parameter vectors is high, we can consider that the precision of the micromotion feature extraction is well, and the forecast of state information in the next scan is accurate. In this case, the searching range gate can be decreased to reduce the computation load. On the contrary, if $C_{s, \mathbf{y}_{k}}$ is relatively large, the forecasted precision is low and the searching range gate should be increased appropriately. Therefore, $f_{R}(\cdot)$ should be an increasing function, which is defined as

$$
f_{R}\left(C_{s, \mathbf{y}_{k}}\right)=r_{m}+\lambda \cdot C_{s, \mathbf{y}_{k}},
$$

where $\lambda$ is a constant coefficient, $r_{m}$ is the minimum of searching range gate, which is set to be 5 in this paper, and the maximum value of $f_{R}(\cdot)$ is limited to 20 .

3.3. Adaptive Starting and Ending of Precession Feature Extraction. In TBD algorithms, the detection threshold affects the target detecting performance directly. However, how to set an appropriate detection threshold is difficult, especially in the multiscatterers environment.

As the micromotion feature extraction has been included into the process of target detecting and tracking by establishing a feedback loop, the pertinent issue now is how to declare the presence of a target and when to start and end the micromotion feature extraction algorithm.

In the proposed method, the energy accumulation value and the result of micromotion feature extraction are both taken into full consideration to address the problem above, 
which is different from the traditional signal processing method. Just as we know, in traditional method, only when the energy accumulation value is larger than the threshold, it declares the presence of a target, and then the extraction of its micromotion feature will be implemented by transmitting additional observation pulses. In comparison, in the proposed method, by adding the micromotion feature extraction into the process of target detecting and tracking, on condition that the precision of micromotion feature extraction is high enough, it can declare the presence of a target and the micromotion feature is extracted successfully at the same time, although the energy accumulation value dose not achieve the required level.

Assume the minimum and maximum total number of scans that are jointly processed in TBD are $K_{N}$ and $K_{M}$, respectively. Firstly, set two detection threshold coefficients: the lower detection threshold coefficient $\gamma_{1}$ and the higher detection threshold coefficient $\gamma_{2}$. In the $k$ th $\left(k \geq K_{N}\right)$ scan if the cumulative energy $I\left(\mathbf{y}_{k}\right)$ is larger than $T_{\alpha_{2}}=\gamma_{2} \cdot k \cdot P$, we declare the presence of a target and the micromotion feature parameters can be obtained with the proposed method described in Section 3.1. On the other hand, if the cumulative energy $I\left(\mathbf{y}_{k}\right)$ is larger than $T_{\alpha_{1}}=\gamma_{1} \cdot k \cdot P$ and smaller than $T_{\alpha_{2}}$, backtracking the target scatterers trajectories according to (10) and (14). On this basis, the extracted micromotion feature parameter vector at $k$ th scan can be obtained. Go on to cumulate energy for the data of $k+1$ th scan, and extract the micromotion feature parameters at $k+1$ th scan. The consistency of the extracted micromotion feature parameter vectors $C_{s, \mathbf{y}_{k}}$ can be calculated according to (29). If $C_{s, \mathbf{y}_{k}}$ and fitting error $\widehat{E}_{f, \mathbf{y}_{k}}$ are both relatively small (satisfies $C_{s, \mathbf{y}_{k}}<T_{C}$ and $\widehat{E}_{f, \mathbf{y}_{k}}<T_{E}$ ), we can declare the presence of a target and get the micromotion feature parameters. The energy accumulation is no longer needed. Otherwise, update the state transition set according to (27), and the energy accumulation of the data of $k+2$ th scan is needed. Repeat the steps described above until it satisfies $C_{s, \mathbf{y}_{k}}<T_{C}$ and $\widehat{E}_{f, \mathbf{y}_{k}}<T_{E}$ or $I\left(\mathbf{y}_{k}\right)>T_{\alpha_{2}}$, or it reached the $K_{M}$ th scan. In conclusion, the flowchart of micromotion feature extraction of space target based on TBD is shown in Figure 4.

There are three points should be noticed for the proposed method.

(1) The values of $\gamma_{1}$ and $\gamma_{2}$ are chosen from Monte-Carlo experiments with the given false alarm probability $P_{\mathrm{FA}, \gamma_{1}}$ and $P_{\mathrm{FA}, \gamma_{2}}$ in the case the presence of a target is declared when the energy accumulation value is larger than the threshold. The corresponding detection probabilities are denoted as $P_{D, \gamma_{1}}$ and $P_{D, \gamma_{2}}$. However, the result of micromotion feature extraction is taken into consideration to declare the presence of a target in this paper; thus the final false alarm probability $P_{\mathrm{FA}}$ and detection probability $P_{D}$ obtained from the proposed method are hard to be calculated from a specific expression with the variables of $P_{\mathrm{FA}, \gamma_{1}}, P_{D, \gamma_{1}}, P_{\mathrm{FA}, \gamma_{2}}$, and $P_{D, \gamma_{2}}$. Numerous experiments have shown that $P_{\mathrm{FA}}$ will be a little higher than $P_{\mathrm{FA}, \gamma_{2}}$. Therefore the value of $P_{\mathrm{FA}, \gamma_{2}}$ can be chosen according to the desired false alarm probability, which is set as $P_{\mathrm{FA}, \gamma_{2}}=0.005$ in this paper. The value of $P_{\mathrm{FA}, \gamma_{1}}$ will affect the detection probability and computation load, and it is set as $P_{\mathrm{FA}, \gamma_{1}}=0.5$ in this paper from numerous experiments with the consideration of maximizing the detection probability and minimizing the computation load.

(2) When the value of $P$ is larger than the observable scatterers number, the cumulative energy and the trajectories which are corresponding to the target will not be different from that corresponding to the noise. Thus, the target will not be detected. On the contrary, if the value of $P$ is smaller than the observable scatterers number, only $P$ trajectories can be obtained. Thus, some scatterers trajectories will be lost and the corresponding micromotion feature parameters can not be extracted. Therefore, the value of $P$ should be set as the maximum possible value firstly, which can be preset by experience. Then the value of $P$ decreases gradually until a target is detected and the micromotion feature is extracted or $P=1$.

(3) The computational load of the proposed method is proportional to the search range. Therefore, to reduce the computational load, we assume that target detecting and tracking with narrow-band radar is conducted firstly, and a lower threshold is used to detect the possible targets; then the obtained coarse position and velocity information of targets are utilized to determine the search range of the proposed method.

\section{Simulations}

In this section, some simulations are carried out to verify the efficiency of the proposed algorithm.

Assume that the target center is located at $(0,0,1000) \mathrm{km}$ at the beginning of observation (i.e., $R_{c}=1000 \mathrm{~km}$ ) and the velocity of target is $500 \mathrm{~m} / \mathrm{s}$. The other simulation parameters are the same with those described in Section 2. The geometry of radar and target is illustrated as shown in Figure 5.

The parameters of the TBD procedure are set as follows: $K_{N}=15, K_{M}=30, N_{r}=N_{\theta}=1000, \Delta r=0.05 \mathrm{~m}, \Delta \theta=$ $0.005^{\circ}, R_{0}=1000 \mathrm{~m}, \theta_{0}=0^{\circ}, \phi_{B}=0.15^{\circ}, \varsigma=0.17, T_{C}=0.1$, $T_{E}=2 \cdot \Delta r=0.1 \mathrm{~m}, P_{\mathrm{FA}, \gamma_{1}}=0.5$, and $P_{\mathrm{FA}, \gamma_{2}}=0.005$. First, we consider the problem of the detection threshold coefficient setting. When the energy accumulation value is larger than the threshold, it declares the presence of a target, and then the false alarm probability versus detection threshold coefficient is reported in Figure 6. Therefore, for the given $P_{\mathrm{FA}, \gamma_{1}}=0.5$ and $P_{\mathrm{FA}, \gamma_{2}}=0.005$, the detection threshold coefficients are set as $\gamma_{1}=1$ and $\gamma_{2}=1.3$.

The micromotion feature extraction based on TBD of $P=$ 3 is conducted with SNR $=8 \mathrm{~dB}$ which is added to the HRRP of the target. Due to the fact that Fourier transform with $N_{r}$ $\left(N_{r}=1000\right)$ points is taken to obtain the HRRP, the average received SNR of the original echo is obtained by subtracting $10 \log (1000)=30 \mathrm{~dB}$ from the reported value. After $K_{N}=15$ scans, among all the state sequences whose cumulative energy is larger than $T_{\alpha_{1}}$ and satisfying the condition shown as (14), one state sequence is corresponding to the target, as shown in Figure $7(\mathrm{a})$, and the other state sequences are induced from noise; one of them is shown as Figure 7(b). For convenience, the ordinate value has reduced by a constant of $1 \times 10^{6}$. 


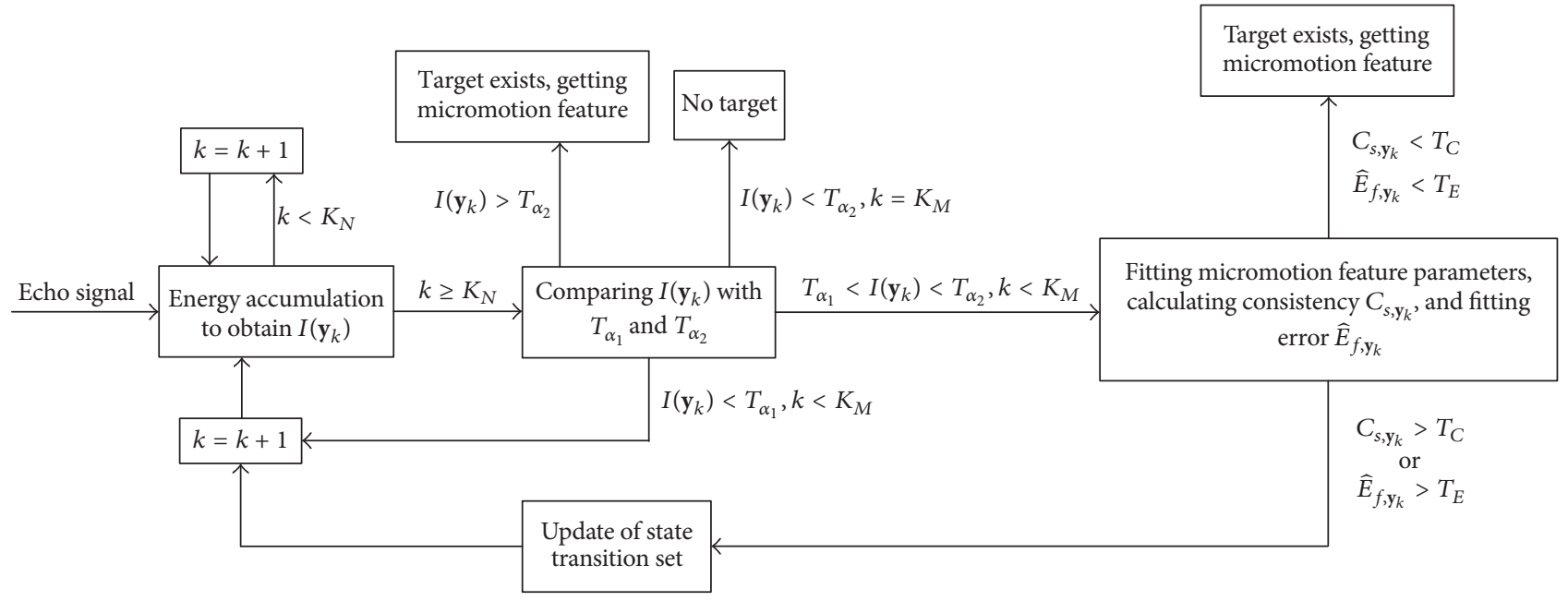

FIGURE 4: Micromotion feature extraction of space target based on TBD.

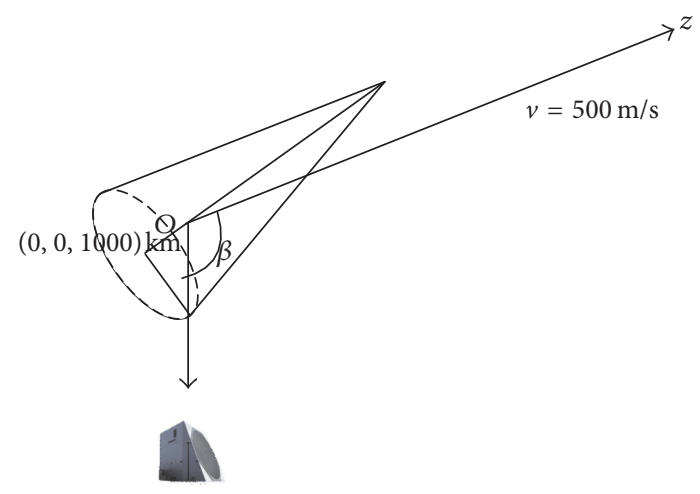

FIGURE 5: Geometry of radar and target.

In the following text, for the state of $\mathbf{y}_{k}$, the cumulative energy, range trajectories, angle trajectories, estimated micromotion feature parameter vector, fitting error, consistency of the extracted micromotion feature parameter vector, state transition set of target, and noise in next scan are denoted as $I_{T}\left(\mathbf{y}_{k}\right), I_{N}\left(\mathbf{y}_{k}\right), R_{\mathbf{y}_{k}, T}, R_{\mathbf{y}_{k}, N}, \Theta_{\mathbf{y}_{k}, T}, \Theta_{\mathbf{y}_{k}, N}, \widehat{\mathbf{P}_{\mathbf{A} f, \mathbf{y}_{k}, T}}, \widehat{\mathbf{P}_{\mathbf{A} f, \mathbf{y}_{k}, N}}$, $\widehat{E}_{f, \mathbf{y}_{k}, T}, \widehat{E}_{f, \mathbf{y}_{k}, N}, C_{s, \mathbf{y}_{k}, T}, C_{s, \mathbf{y}_{k}, N}, \Gamma_{T}\left(\mathbf{y}_{k+1}\right)$, and $\Gamma_{N}\left(\mathbf{y}_{k+1}\right)$, respectively.

Using any two values of the range trajectory, the probable slope can be obtained, which can be utilized for the coarse slope compensation of trajectories, as shown in Figure 8.

Based on the range trajectories of target and noise, respectively, the estimated micromotion feature parameter vectors $\widehat{\mathbf{P}}_{\mathbf{A} f, \mathbf{y}_{k}, T}$ and $\widehat{\mathbf{P}}_{\mathbf{A} f, \mathbf{y}_{k}, N}$ can be obtained according to (13)-(22). At this time, the energy accumulation values $I_{T}\left(\mathbf{y}_{k}\right)$ and $I_{N}\left(\mathbf{y}_{k}\right)$ are both smaller than threshold $T_{\alpha_{2}}$, and the consistencies $C_{s, \mathbf{y}_{k}, T}$ and $C_{s, \mathbf{y}_{k}, N}$ can not be obtained due to the inexistence of the extracted micromotion feature parameter vector at the former scan. Therefore, it is necessary to continue to carry out energy accumulation. For $I_{T}\left(\mathbf{y}_{k}\right)$ and $I_{N}\left(\mathbf{y}_{k}\right)$, respectively, update state transition sets $\Gamma_{T}\left(\mathbf{y}_{k+1}\right)$

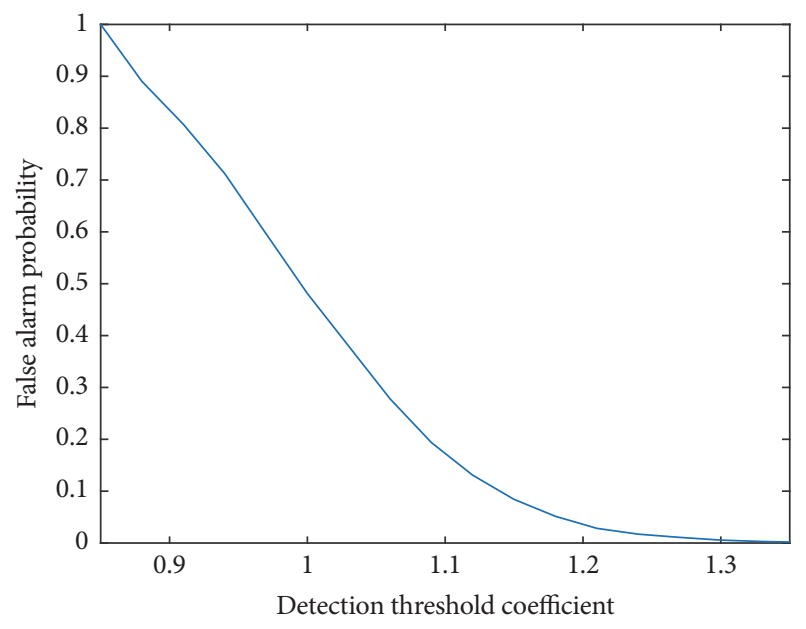

FIGURE 6: False alarm probability versus detection threshold coefficient.

and $\Gamma_{N}\left(\mathbf{y}_{k+1}\right)$ according to (27) by utilizing the obtained precession feature parameter vectors $\widehat{\mathbf{P}}_{\mathbf{A} f, \mathbf{y}_{k}, T}$ and $\widehat{\mathbf{P}_{\mathbf{A}} f \mathbf{y}_{k}, N}$.

After 24 scans, for $I_{N}\left(\mathbf{y}_{k}\right)$, we can obtain the consistency $C_{s, \mathbf{y}_{k}, N}=3.92$ and fitting error $\widehat{E}_{f, \mathbf{y}_{k}, N}=0.26$, which does not satisfy $C_{s, \mathbf{y}_{k}}<T_{C}$ and $\widehat{E}_{f, \mathbf{y}_{k}}<T_{E}$. On the contrary, for $I_{T}\left(\mathbf{y}_{k}\right)$, the consistency $C_{s, \mathbf{y}_{k}, T}=0.08$ and fitting error $\widehat{E}_{f, \mathbf{y}_{k}, T}=$ 0.05 , which satisfies $C_{s, y_{k}}<T_{C}$ and $\widehat{E}_{f, y_{k}}<T_{E}$. Therefore, we declare the presence of a target, and the micromotion feature parameter vector $\widehat{\mathbf{P}}_{\mathbf{A} f, \mathbf{y}_{k}, T}$ can be obtained, as shown in Table 1. And the update process of the consistency, fitting error, and the size of searching range gate are shown in Figure 9.

From Figure 8, we can see that the consistency of the extracted micromotion feature parameter vector is increasing (i.e., $C_{s, \mathbf{y}_{k}}$ is decreasing) with energy accumulation, and $C_{s, \mathbf{y}_{k}, T}$ can reduce to the value smaller than $T_{C}=0.1$, while $C_{s, y_{k}, N}$ is always higher than $T_{C}=0.1$. Similarly, the size 


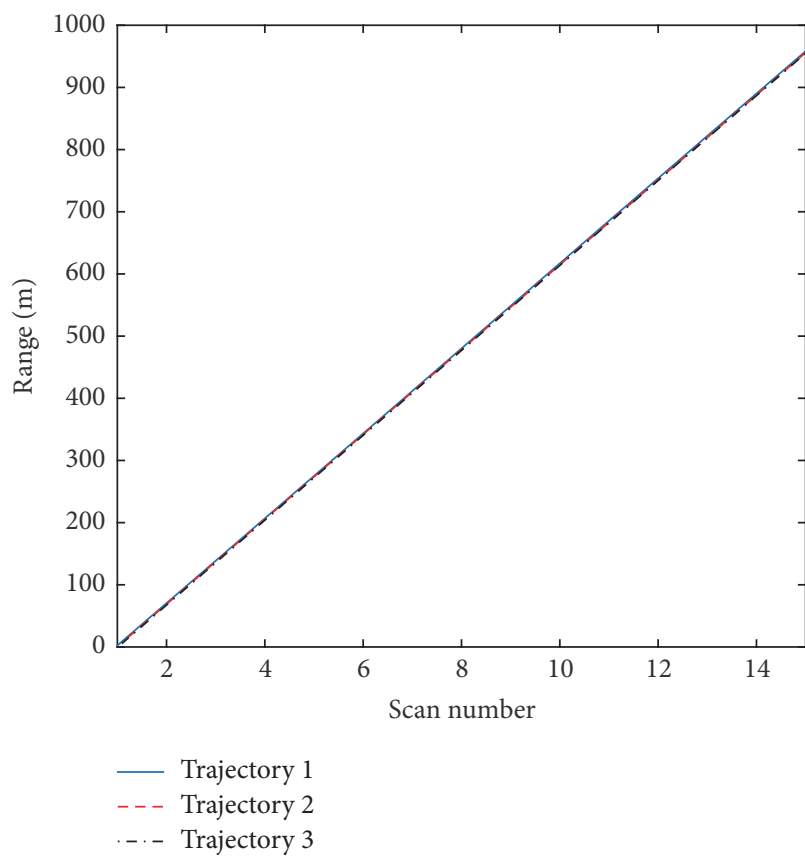

(a)

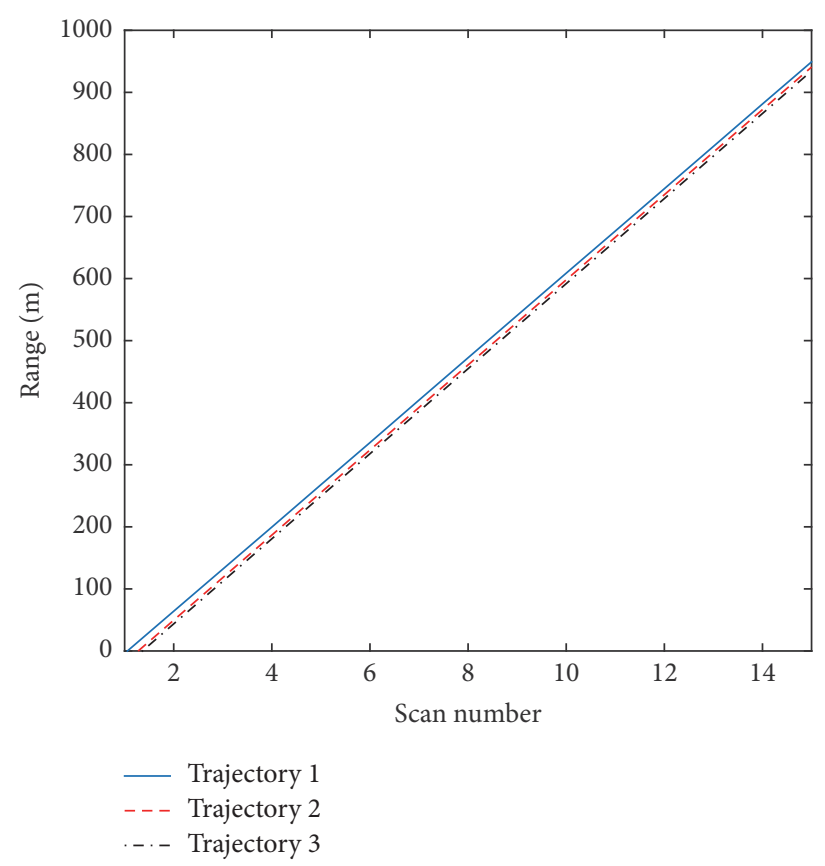

(b)

FIGURE 7: Backtracked range trajectories: (a) induced by target; (b) induced by noise.

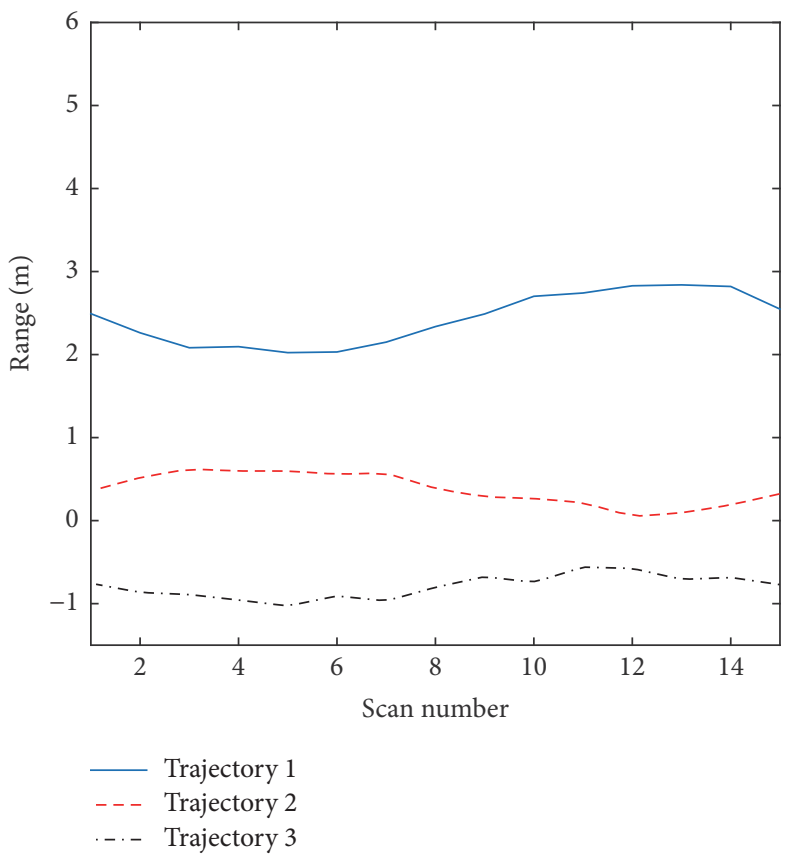

(a)

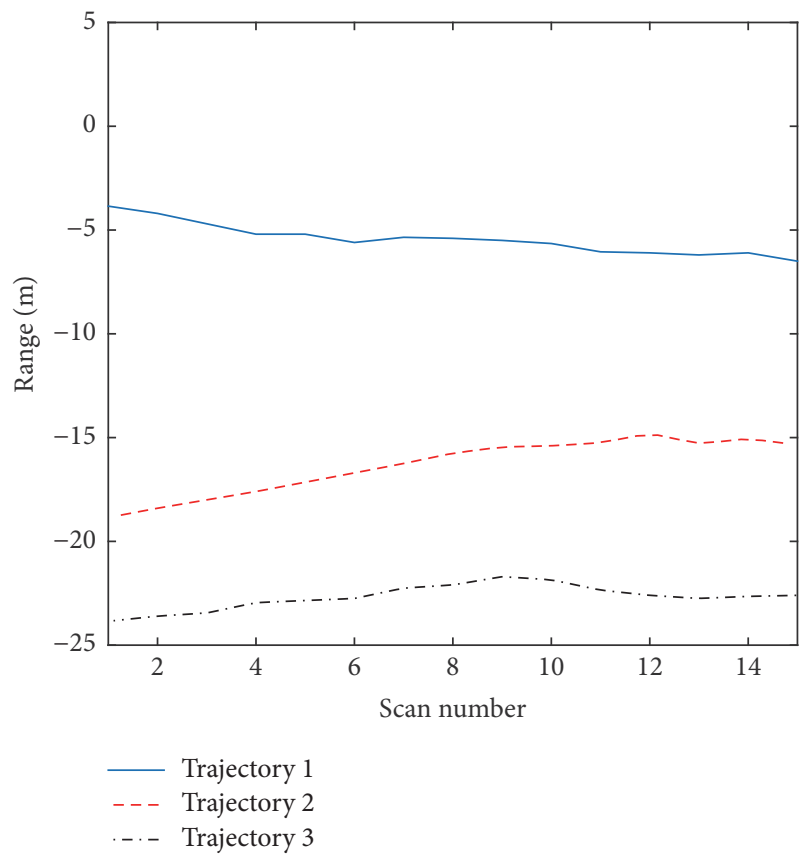

(b)

FIGURE 8: Backtracked range trajectories with coarse slope compensation; (a) induced by target; (b) induced by noise.

of searching range gate has the same change trend with the consistency. At the same time, the fitting error increases with energy accumulation, and $\widehat{E}_{f, \mathbf{y}_{\mathrm{k}}, T}$ can stabilize at the value which is smaller than $T_{E}$, while $\widehat{E}_{f, y_{k}, N}$ will be higher than $T_{E}$. Therefore, the state sequence corresponding to the target can be selected out, and the other state sequences induced from noise can be eliminated. Furthermore, the obtained micromotion feature parameter vector shown in Table 1 is closed to the theoretical value, which illustrates the effectiveness of the proposed method. 

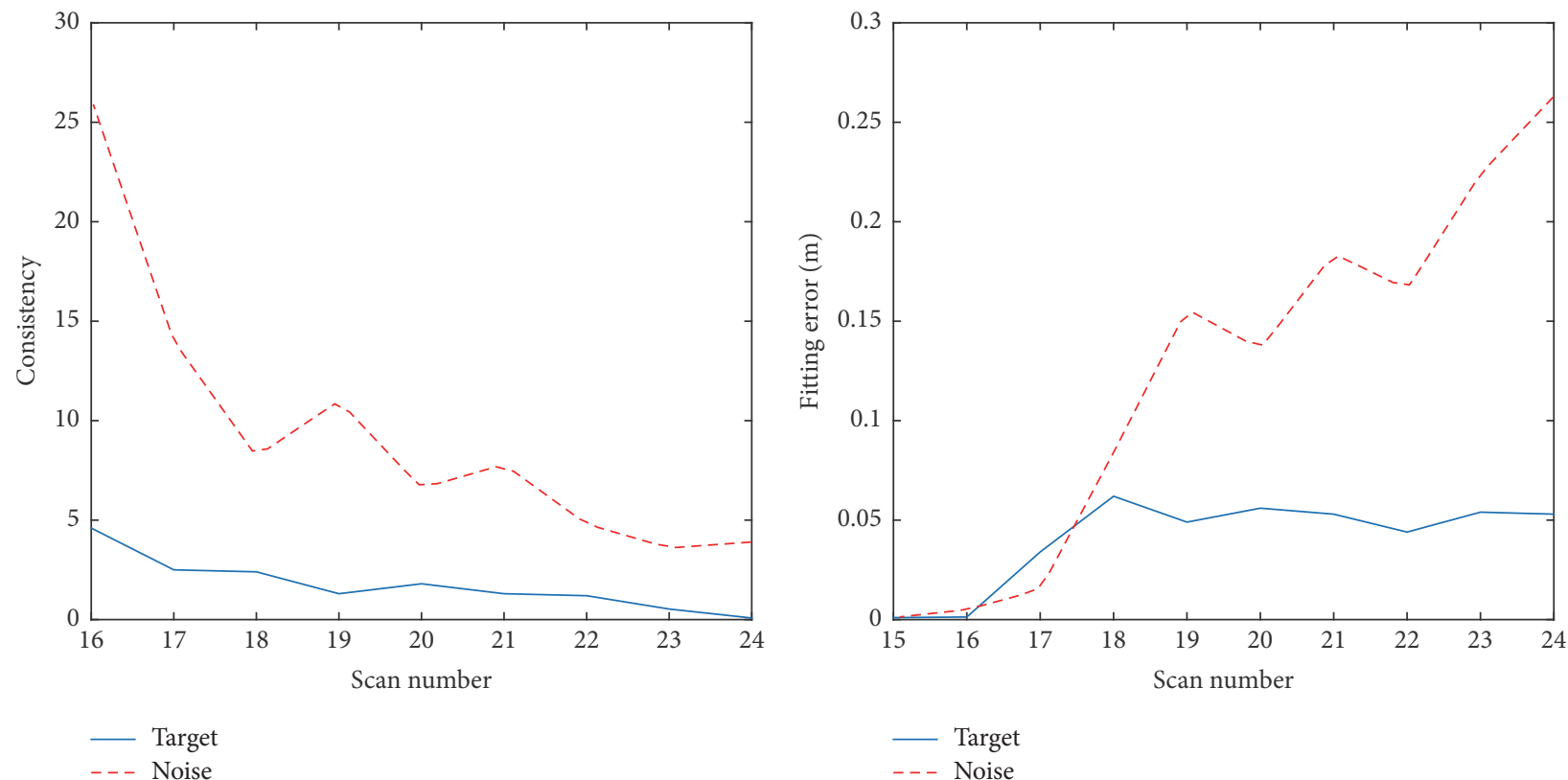

(a)

(b)

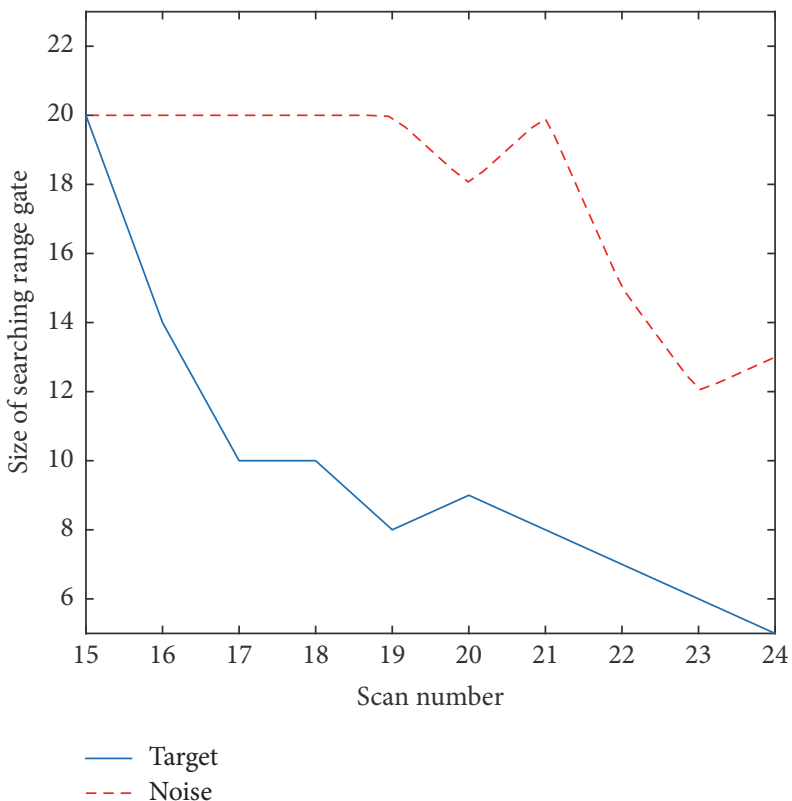

(c)

FIgURE 9: Update process. (a) Consistency of the extracted micromotion feature parameters. (b) Fitting error. (c) Size of searching range gate.

TABLE 1: Extracted micromotion feature parameter vector.

\begin{tabular}{lccccccccc}
\hline Parameter & $\begin{array}{c}|o a| \\
(\mathrm{m})\end{array}$ & $\begin{array}{c}|o b| \\
(\mathrm{m})\end{array}$ & $\begin{array}{c}r_{0} \\
(\mathrm{~m})\end{array}$ & $\begin{array}{c}\phi_{0} \\
(\mathrm{rad})\end{array}$ & $\begin{array}{c}\beta \\
\left(^{\circ}\right)\end{array}$ & $\begin{array}{c}9 \\
\left({ }^{\circ}\right)\end{array}$ & $\begin{array}{c}\omega \\
(\mathrm{rad} / \mathrm{s})\end{array}$ & $\begin{array}{c}v \\
(\mathrm{~m} / \mathrm{s})\end{array}$ & $\begin{array}{c}R_{c} \\
(\mathrm{~m})\end{array}$ \\
\hline $\begin{array}{l}\text { Ture value } \\
\text { Estimated }\end{array}$ & 3.000 & 0.300 & 1.000 & 0.031 & 2.530 & 0.261 & 25.132 & 500.000 & $10^{6}$ \\
$\begin{array}{l}\text { value } \\
\text { Error (\%) }\end{array}$ & 2.892 & 0.319 & 0.911 & 0.028 & 2.625 & 0.252 & 26.013 & 492.114 & $1.000023 \times 10^{6}$ \\
& 3.60 & 6.33 & 8.90 & 9.67 & 3.75 & 3.45 & 3.52 & 1.58 & 0.00 \\
\hline
\end{tabular}


TABLE 2: Extracted micromotion feature parameter vector in with extended Hough transform method.

\begin{tabular}{lccccccccc}
\hline Parameter & $\begin{array}{c}|o a| \\
(\mathrm{m})\end{array}$ & $\begin{array}{c}|o b| \\
(\mathrm{m})\end{array}$ & $\begin{array}{c}r_{0} \\
(\mathrm{~m})\end{array}$ & $\begin{array}{c}\phi_{0} \\
(\mathrm{rad})\end{array}$ & $\begin{array}{c}\beta \\
\left.{ }^{\circ}\right)\end{array}$ & $\begin{array}{c}9 \\
\left(^{\circ}\right)\end{array}$ & $\begin{array}{c}\omega \\
(\mathrm{rad} / \mathrm{s})\end{array}$ & $\begin{array}{c}v \\
(\mathrm{~m} / \mathrm{s})\end{array}$ & $\begin{array}{c}R_{c} \\
(\mathrm{~m})\end{array}$ \\
\hline $\begin{array}{l}\text { Ture value } \\
\text { Estimated }\end{array}$ & 3.000 & 0.300 & 1.000 & 0.031 & 2.530 & 0.261 & 25.132 & 500.000 \\
$\begin{array}{l}\text { value } \\
\text { Error (\%) }\end{array}$ & 6.200 & 0.500 & 0.600 & 0.100 & 1.700 & 0.100 & 13.800 & 653.000 & $1.000043 \times 10^{6}$ \\
& 106.67 & 66.67 & 40.00 & 222.58 & 32.81 & 61.69 & 45.09 & 30.60 \\
\hline
\end{tabular}

Next, the detection performance and micromotion feature extraction performance of the proposed method are compared with the traditional TBD method and the extended Hough transform method, respectively. With the proposed method, the false alarm probability and the detection probability are $P_{\mathrm{FA}}=0.0052$ and $P_{D}=0.8823$. With the traditional TBD method, fixing the false alarm probability as 0.0052 , the detection probability is 0.4827 . Obviously, the detection performance of the proposed method is better than that of the traditional TBD method. Further, the micromotion feature extraction performance is defined as the estimated error of each micromotion feature parameter. With the extended Hough transform method, the micromotion feature parameter vector is obtained shown in Table 2. Due to the low SNR, the micromotion feature parameter vector can not be extracted effectively with the extended Hough transform method, while the high micromotion feature extraction performance can be obtained with the proposed method, shown as Table 1.

Finally, the detection performance and micromotion feature extraction performance with different SNRs are considered. Fixing the false alarm probability as 0.0052 , the detection probability of the proposed method is better than that of the traditional TBD method, shown in Figure 10. Without loss of generality, we think the micromotion feature extraction is successful when the estimated error of each micromotion feature parameter is less than $10 \%$. The success of micromotion feature extraction with different SNRs is shown in Figure 11. We can see that the success of micromotion feature extraction with the proposed method is much higher than that with the extended Hough transform method when SNR is lower than $11 \mathrm{~dB}$. What is more important is that the micromotion feature parameters can be extracted without transmitting additional pulses, which can save the radar resources and provide real-time information for target recognition.

\section{Conclusions}

A micromotion feature extraction method for space target based on TBD is proposed in this paper. This method establishes a feedback loop between micromotion feature extraction and TBD of target. As a result, the micromotion feature can be extracted concurrent with target detecting and tracking, and the detection performance can be improved. The steps of the method are described in detail, and some simulations are given to illustrate its effectiveness. It is noted that the cone-shaped target is taken as an example

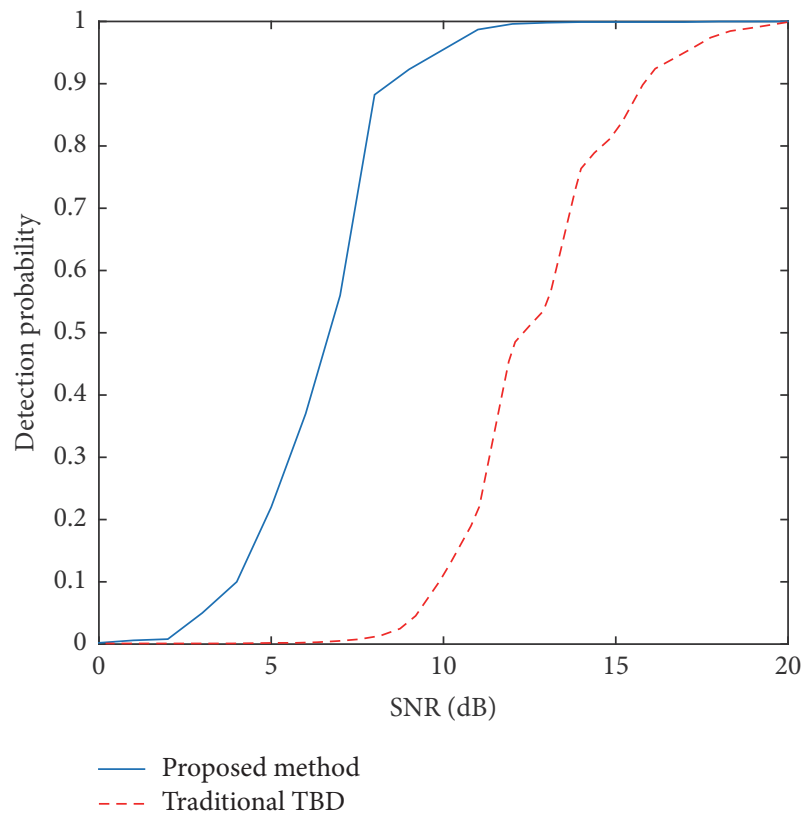

FIGURE 10: Detection probability versus SNR.

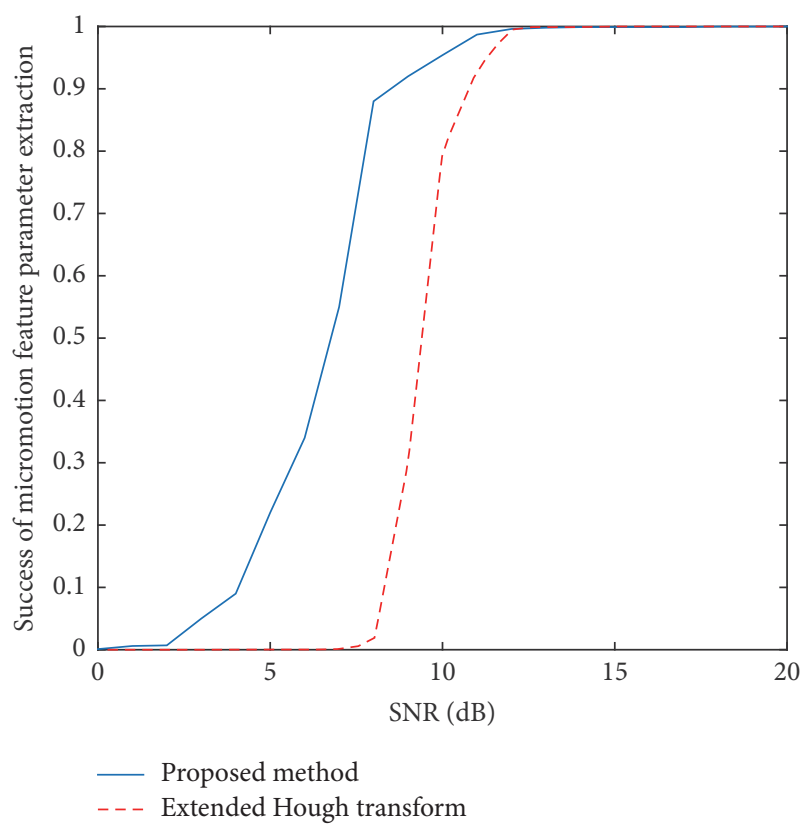

FIGURE 11: Success of micromotion feature extraction versus SNR. 
for describing and verifying the proposed feature extracting method. In fact, the proposed method is not influenced by the shape of the target. The proposed feature extracting method is suitable for the fixed scatterer model, slide scatterer model, and any other kinds of micromotion forms by using the corresponding fitting curves.

However, the radar resource is limited; the resource saturation will be an important problem when there are many targets coexisting in the radar monitoring area. Thus, the reasonable and effective resources scheduling algorithms are important for exploiting the benefits of the proposed method. The related study will be presented in another independent manuscript.

\section{Conflicts of Interest}

The authors declare that there are no conflicts of interest regarding the publication of this article.

\section{Acknowledgments}

This work was supported in part by the National Natural Science Foundation of China under Grants 61631019, 61471386, and 61571457 and in part by Ministry of Education, Singapore, under Grant MOE2016-T2-1-070.

\section{References}

[1] Y. Luo, Q. Zhang, N. Yuan, F. Zhu, and F. Gu, "Three-dimensional precession feature extraction of space targets," IEEE Transactions on Aerospace and Electronic Systems, vol. 50, no. 2, pp. 1313-1329, 2014.

[2] P. Suresh, T. Thayaparan, T. Obulesu, and K. Venkataramaniah, "Extracting micro-doppler radar signatures from rotating targets using fourier-bessel transform and time-frequency analysis," IEEE Transactions on Geoscience and Remote Sensing, vol. 52, no. 6, pp. 3204-3210, 2014.

[3] B. Peng, Z. Liu, X. Wei, and X. Li, "Sinusoidal Frequency Modulation Sparse Recovery for Precession Rate Estimation Using Low-Frequency Long-Range Radar," IEEE Sensors Journal, vol. 15, no. 12, pp. 7329-7340, 2015.

[4] V. C. Chen, F. Li, S.-S. Ho, and H. Wechsler, "Micro-doppler effect in radar: Phenomenon, model, and simulation study," IEEE Transactions on Aerospace and Electronic Systems, vol. 42, no. 1, pp. 2-21, 2006.

[5] X. Chen, J. Guan, X. Li, and Y. He, "Effective coherent integration method for marine target with micromotion via phase differentiation and radon-Lv's distribution," IET Radar, Sonar and Navigation, vol. 9, no. 9, pp. 1284-1295, 2015.

[6] J.-B. Zhuang, Z.-M. Deng, Y.-S. Ye, Y.-X. Zhang, and Y.-Y. Chen, "Micro-doppler ambiguity resolution based on shorttime compressed sensing," Journal of Electrical and Computer Engineering, vol. 2015, Article ID 864508, 2015.

[7] Y.-X. Liu, X. Li, and Z.-W. Zhuang, "Estimation of micromotion parameters based on micro-Doppler," IET Signal Processing, vol. 4, no. 3, pp. 213-217, 2010.

[8] J. Niu, K. Li, W. Jiang, X. Li, G. Kuang, and H. Zhu, "A new method of micro-motion parameters estimation based on cyclic autocorrelation function," Science China Information Sciences, vol. 56, no. 10, pp. 1-11, 2013.
[9] C. Cai, W. Liu, J. S. Fu, and L. Lu, "Empirical mode decomposition of micro-Doppler signature," in Proceedings of the 2005 IEEE International Radar Conference Record, RADAR 2005, pp. 895-899, usa, May 2005.

[10] Y. Luo, Q. Zhang, C. W. Qiu, S. Li et al., "Micro-Doppler feature extraction for wideband imaging radar based on complex image orthogonal matching pursuit decomposition," IET Radar, Sonar, and Navigation, vol. 7, no. 8, pp. 914-924, 2013.

[11] M. Li and Y. Jiang, "Bistatic occlusion effect of missile warhead based on micro-Doppler effect," Optik-International Journal for Light and Electron Optics, vol. 125, no. 19, pp. 5630-5634, 2014.

[12] L. Ma, J. Liu, T. Wang, Y. Li, and X. Wang, "Micro-Doppler characteristics of sliding-type scattering center on rotationally symmetric target," Science China. Information Sciences, vol. 54, no. 9, pp. 1957-1967, 2011.

[13] X. Bai and Z. Bao, "High-resolution 3D imaging of precession cone-shaped targets," IEEE Transactions on Antennas and Propagation, vol. 62, no. 8, pp. 4209-4219, 2014.

[14] X. Bai and Z. Bao, "Imaging of rotation-symmetric space targets based on electromagnetic modeling," IEEE Transactions on Aerospace and Electronic Systems, vol. 50, no. 3, pp. 1680-1689, 2014.

[15] X. Pan, W. Wang, J. Liu, D. Feng, Y. Liu, and G. Wang, "Features extraction of rotationally symmetric ballistic targets based on micro-Doppler," Progress in Electromagnetics Research, vol. 137, pp. 727-740, 2013.

[16] J. Yan, H. Liu, B. Jiu, Z. Liu, and Z. Bao, "Joint Detection and Tracking Processing Algorithm for Target Tracking in Multiple Radar System," IEEE Sensors Journal, vol. 15, no. 11, pp. 65346541, 2015.

[17] S. M. Tonissen and R. J. Evans, "Performance of dynamic programming techniques for track-before-detect," IEEE Transactions on Aerospace and Electronic Systems, vol. 32, no. 4, pp. 1440-1451, 1996.

[18] F. Papi, V. Kyovtorov, R. Giuliani, F. Oliveri, and D. Tarchi, "Bernoulli filter for track-before-detect using MIMO radar," IEEE Signal Processing Letters, vol. 21, no. 9, pp. 1145-1149, 2014.

[19] Y.-J. Chen, Q. Zhang, H. Jiang, Y. Luo, and Y.-A. Chen, "A cognitive feature extracting method for space target," in Proceedings of the 36th IEEE International Geoscience and Remote Sensing Symposium, IGARSS 2016, pp. 3148-3151, chn, July 2016.

[20] S. Buzzi, M. Lops, L. Venturino, and M. Ferri, “Track-beforedetect procedures in a multi-target environment," IEEE Transactions on Aerospace and Electronic Systems, vol. 44, no. 3, pp. 1135-1150, 2008.

[21] H. Jiang, W. Yi, G. Cui, L. Kong, and X. Yang, "Trackbefore-detect strategies for range distributed target detection in compound-Gaussian clutter," Signal Processing, vol. 120, pp. 462-467, 2016.

[22] M. Zhao, Q. Zhang, Y. Luo, and L. Sun, "Micromotion Feature Extraction and Distinguishing of Space Group Targets," IEEE Geoscience and Remote Sensing Letters, vol. 14, no. 2, pp. 174178, 2017.

[23] G. Dartmann, E. Zandi, and G. Ascheid, "A modified levenbergmarquardt method for the bidirectional relay channel," IEEE Transactions on Vehicular Technology, vol. 63, no. 8, pp. 40964101, 2014.

[24] X. Bai, M. Xing, F. Zhou, G. Lu, and Z. Bao, "Imaging of micromotion targets with rotating parts based on empiricalmode decomposition," IEEE Transactions on Geoscience and Remote Sensing, vol. 46, no. 11, pp. 3514-3523, 2008. 


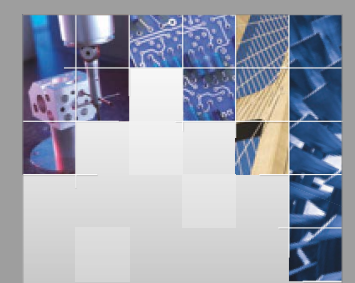

\section{Enfincering}
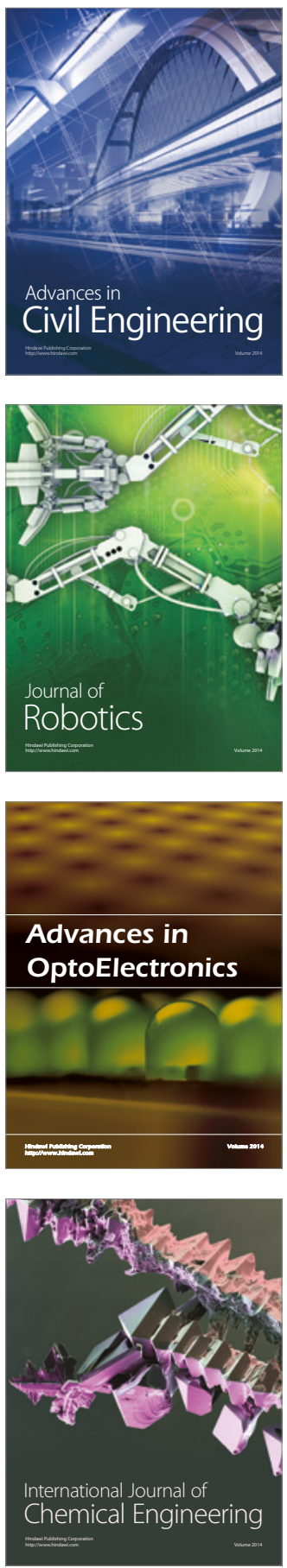

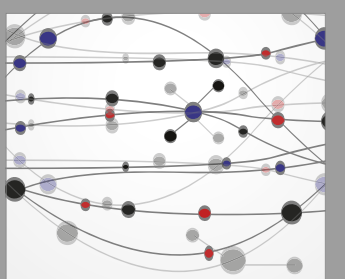

The Scientific World Journal

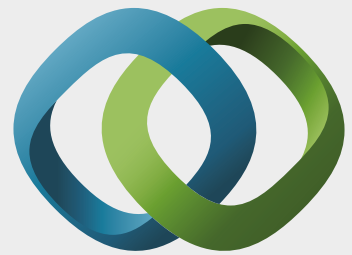

\section{Hindawi}

Submit your manuscripts at

https://www.hindawi.com
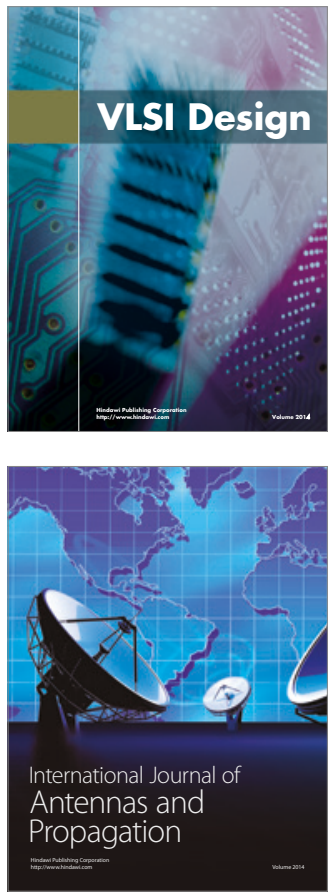

\section{Rotating}

Machinery
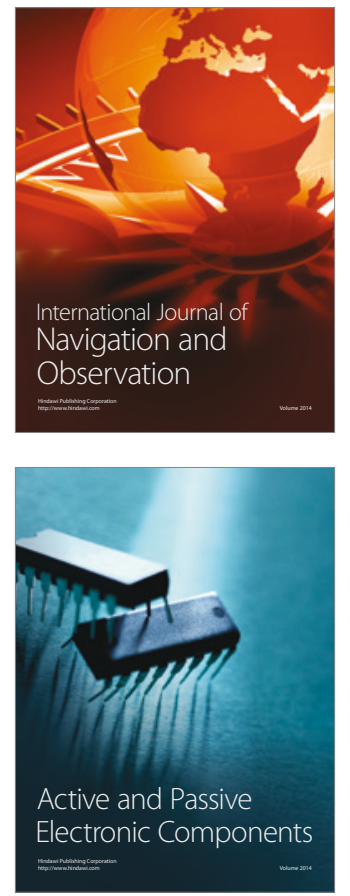
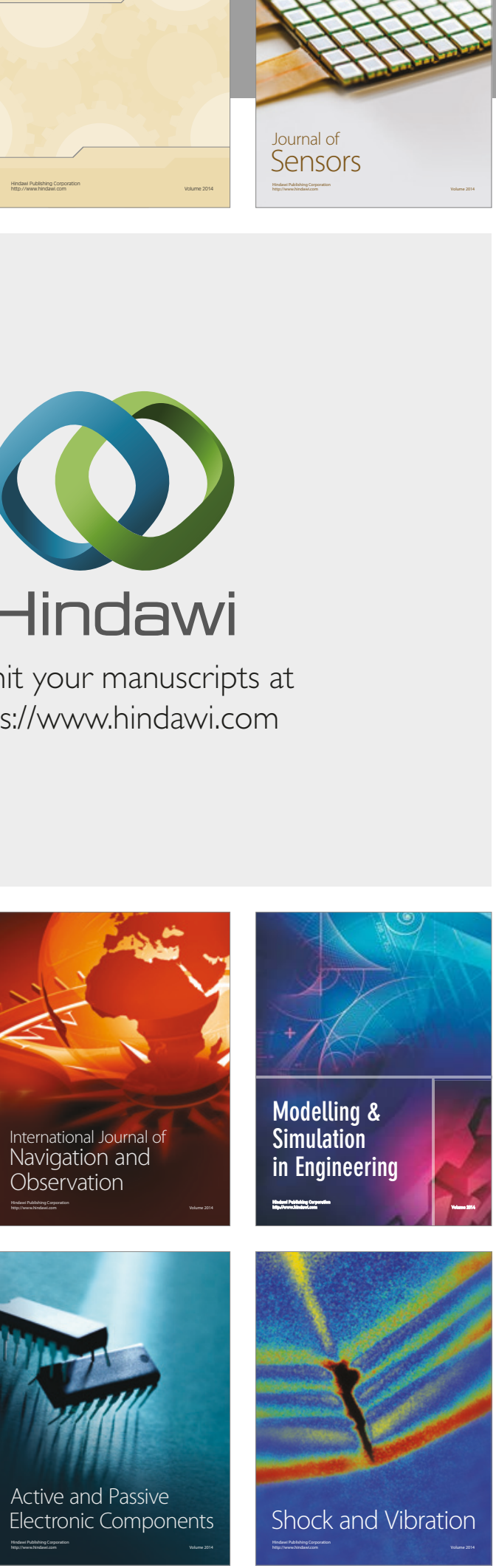
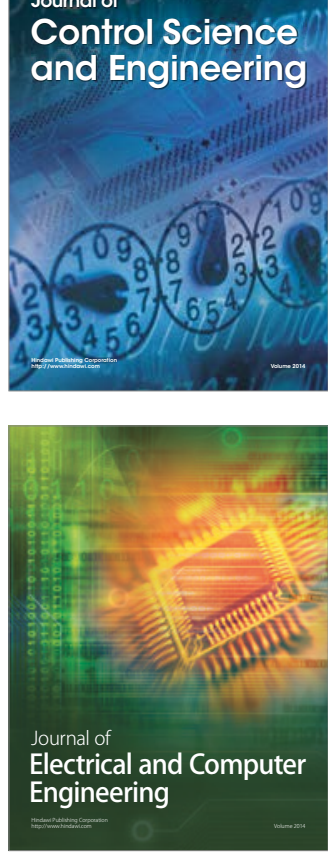

Distributed

Journal of

Control Science

and Engineering
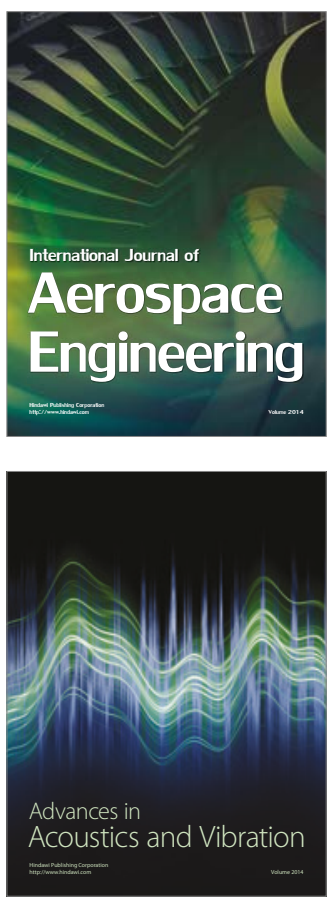

Sensor Networks 